\title{
The Valuation of Caps, Floors and Swaptions in a Multi-Factor Spot-Rate Model ${ }^{1}$
}

\author{
Sandra Peterson ${ }^{2} \quad$ Richard C. Stapleton ${ }^{3} \quad$ Marti G. Subrahmanyam ${ }^{4}$
}

First draft: April, 1998

This draft: October 3, 2001

\begin{abstract}
${ }^{1}$ We thank V. Acharya and P. Pasquariello for able research assistance, and Q. Dai and S. Das for helpful comments on earlier drafts. We acknowledge comments on earlier versions of this paper by participants in seminars at the University of Cardiff, University of London, Birkbeck College, The Australian Graduate School of Management, Sydney, the Fields Institute, Toronto, UCLA and Temple University. We are also grateful for discussion of the paper at several conferences, including those of the European Finance Association, the European Financial Management Association, the Scottish Institute for Research in Investment and Finance and the Global Association of Risk Professionals. We would also like to thank the editor, B. Dumas, and an anonymous referee, whose detailed comments lead to considerable improvements in the paper.

${ }^{2}$ Scottish Institute for Research in Investment and Finance, Strathclyde University, Glasgow, UK. Tel:(44)141-548-4958, e-mail:s.peterson@telinco.co.uk

${ }^{3}$ Department of Accounting and Finance, Strathclyde University, Glasgow, UK. Tel:(44)1524381172, Fax:(44)524-846 874, e-mail:rcs@staplet.demon.co.uk and University of Melbourne, Australia

${ }^{4}$ Leonard N. Stern School of Business, New York University, Management Education Center, 44 West 4th Street, Suite 9-190, New York, NY10012-1126, USA. Tel: (212)998-0348, Fax: (212)9954233, e-mail:msubrahm@stern.nyu.edu
\end{abstract}




\section{Abstract \\ The Valuation of Caps, Floors and Swaptions in a Multi-Factor Spot-Rate Model.}

We build a multi-factor, no-arbitrage model of the term structure of spot interest rates. The stochastic factors are the short-term interest rate and the premia of the futures rates over the short-term interest rate. In the three-factor version of the model, for example, the first factor is the three-month $L I B O R$, the second factor is the premium of the first futures $\angle I B O R$ over spot $L I B O R$, and the third factor is the incremental premium of the second futures over the first. The model provides an extension of the lognormal interest rate model of Black and Karasinski (1991) to multiple factors, each of which can exhibit mean-reversion.

The method is computationally efficient for several reasons. First, we suggest calibrating the model to $L I B O R$ futures prices, which enables us to can satisfy the no-arbitrage condition without resorting to iterative methods. Second, we modify and implement the binomial approximation methodology of Nelson and Ramaswamy (1990) and Ho, Stapleton and Subrahmanyam (1995) to compute a multi-period tree of rates with the no-arbitrage property. The method uses a recombining two or three-dimensional binomial lattice of interest rates that minimizes the number of states and term structures over time. In addition to these computational advantages, a key feature of the model is that it is consistent with the observed term structure of futures rates as well as the term structure of volatilities implied by the prices of interest rate caps and floors. We use the model to price European-style and Bermudan-style swaptions and yield-spread options.

To implement the methodology, we first calibrate the model to the caplet implied-volatility curve on a given day, and then use the model to price European-style swaptions. We find that the two-factor model, where the LIBOR mean reverts rapidly to a slowly meanreverting second factor, overprices the swaptions relative to market quotations. However, introducing a third factor significantly reduces the overpricing. The calibrated model is used to price Bermudan-style swaptions and yield-spread options. Then, we re-calibrate the twofactor model simultaneously to caplet and swaption prices and use the model output to price Bermudan-style swaptions. 


\section{Introduction}

Satisfactory models exist for the pricing of interest-rate dependent derivatives in a singlefactor context, where interest rates of various maturities are perfectly correlated. For example, assuming that the short-term interest rate follows a mean-reverting process, Jamshidian (1989) prices options on coupon bonds using an extension of the Vasicek (1977) model. Also, assuming a lognormal process, Black, Derman and Toy (1990) and Black and Karasinski (1991) use a binomial tree of interest rates to price interest-rate derivatives. However, these models, by definition, are not capable of accurately pricing derivatives, such as swaptions and yield-spread options, whose payoffs are sensitive to the shape as well as the level of the term structure. In principle, these options require at least a two-factor model of the interest rate process for pricing and hedging. ${ }^{1}$

One promising approach, used extensively in recent work, has been to build no-arbitrage, multi-factor forward-rate models of the Heath, Jarrow and Morton (1992) (HJM) type. In practical applications, these usually take the form of the London Interbank Offer Rate ( $L I$ BOR) based market model of Brace, Gatarek and Musiela, (1997) (BGM) and Miltersen, Sandmann and Sondermann (1997) (MSS). However, this forward-rate approach has some drawbacks for the pricing of swaptions and American-style claims. Most tractable applications require restrictive assumptions on the volatility structure of the forward rates to ensure that the Markov property is satisfied, and for the resulting model to be computable for realistic examples. Hence, while in principle, the forward-rate approach provides a solution, in practice, it is difficult to implement except for certain special cases. $^{2}$

In this paper, we present an alternative, spot-rate model in which LIBOR follows a process with a stochastic central tendency. This is in line with the term structure models pioneered by Hull and White (1994), Jegadeesh and Pennacci (1997), Balduzzi, Das and Foresi (1998)and reviewed by Dai and Singleton (2000). However, we assume that the process for $L I B O R$ is lognormal under the risk neutral measure. We then derive the no-arbitrage restrictions for such a model. Since we assume that the LIBOR rate is lognormal and mean-reverting, our model can also be seen as a multi-factor extension of the Black and

\footnotetext{
${ }^{1}$ For a critique of existing methods for the valuation of swaptions, see Longstaff, Santa-Clara and Schwartz (2001). Of course, one-factor models are adequate for the valuation of European-style options on the shortterm interest rate, such as interest-rate caps and floors.

${ }^{2}$ Ritchken and Sankasubramanian (1995) identify necessary and sufficient conditions on the volatility structure required in order to capture the path dependence in a single state variable. Li, Ritchken and Sankasubramanian (1995) implement this one factor, two state-variable model and price American-style interest rate claims. Alternatively, the pricing of American-style and Bermudan-style claims requires an approximation of the early exercise decision, as detailed in Anderson (2000) and discussed in Anderson and Andreasen (2001).
} 
Karasinski (1991) (BK) model. We illustrate the model using realistic examples with a large number of time periods. We show that it is easy to calibrate the model to the observed cap and swaption prices as well as to the current term structure of futures rates. In the twofactor case, the computational efficiency is achieved through the use of a two-dimensional recombining lattice of interest rates. ${ }^{3}$

Since recent models for the pricing of Bermudan-style and exotic options have taken the BGM-MSS market model approach, it is important to distinguish our model from this class of models. The main difference is the assumption of lognormal LIBORs. In the market model, $L I B O R$ is assumed to be lognormal under the $T+1$ period forward measure. It is not lognormal under the period-by-period risk neutral measure. In our model, as in $\mathrm{BK}$, it is assumed that $L I B O R$ is lognormal under the period-by-period risk neutral measure. Hence, out-of-the-money caplets and swaptions will have different prices in our model than in the market model, even when the models are calibrated to the same market data. ${ }^{4}$ A second difference arises in the pricing of Bermudan-style claims. In order to price these claims we require the process for the spot rate. In our model this is directly modelled. However, in the market model, the spot rate process has to be derived from the forward rate process. In doing this, simplifying assumptions are usually made, which will produce pricing differences. Further, it is clear from the work of Rebonato (1999) and Longstaff, Santa-Clara and Schwartz (2001) that the pricing of swaptions in the market model requires an exogenous specification of the correlation matrix of forward rates. The important difference in our model is that the correlation of forward rates is restricted by the parameters of the stochastic central tendency model of the spot LIBOR. Hence, we would again expect different prices of swaptions in our spot-rate model.

In the calibration of the model, we take as given the prices (or equivalently, the implied volatilities) of European-style interest rate caplets. The problem, as in Black, Derman and Toy (1990) and Black and Karasinski (1991), is to price European-style, Bermudan-style and American-style swaptions, as well as more complex instruments such as yield-spread options, given the prices of the caplets. We also introduce an alternative calibration, where the model is fitted to both caplets and European-style swaptions. This version of the model is also used to price Bermudan-style swaptions.

The computational method introduced to approximate the model builds on previous work by Nelson and Ramaswamy (1990) and Ho, Stapleton and Subrahmanyam (1995) (HSS).

\footnotetext{
${ }^{3}$ Also, since the model is calibrated to the given term structure of futures rates, we avoid the use of iterative methods normally used to calibrate models to the current term structure

${ }^{4}$ This could be an advantage of our model, since market quotes for out-of-the-money caplets suggest that the $L I B O R$ market model assumptions are not consistent with the data. Recent work on forward rate models has made alternative assumptions in order to try to capture the smile.
} 
Nelson and Ramaswamy approximate a single-variable diffusion with a "simple" binomial tree, i.e., a binomial tree with the recombining node property. HSS extend this method to multiple, correlated variables in the case of log-normal diffusion processes. In the context of a two-factor interest rate model, preservation of the no-arbitrage condition in a simple bivariate tree requires a further modification of this methodology. In our model, expectations of subsequent spot rates are determined by the futures rate. In a modification of the HSS method, we capture this dependence, and hence the no-arbitrage property, in a nonexploding tree structure, by allowing the probabilities of moving up or down to depend upon the outcomes of both the spot and the futures LIBOR.

The outline of the paper is as follows. Section 2 reviews the literature on term-structure models and their relationship to the model developed here. Section 3 presents the multifactor model, derives its no-arbitrage properties, and discusses its input requirements. Section 4 derives the methodology for approximating the multi-dimensional diffusion process for the spot LIBOR. Section 5 presents examples of the output of the two-factor model and discusses the computational efficiency of the methodology. In section 6 we show how the model can be calibrated to cap/floor and/or swaption implied volatilities. We then illustrate how the model can be used to value Bermudan-style swaptions and discuss possible extensions to other interest rate derivatives. Section 7 concludes with a discussion of the remaining issues of empirical parameter estimation, and possible extensions of the research.

\section{Term-structure Models}

In early attempts to value interest rate options, Brennan and Schwartz (1979) and Courtadon (1982) derive equilibrium models of the term structure along the lines of the Vasicek (1977) model. However, since the contribution of Ho and Lee (1986), it has been recognized that interest rate dependent claims can be priced within a no-arbitrage model. Hull and White (1994), for example, develop an extended Vasicek model in which interest rates, under the risk-neutral measure, are Gaussian, and exactly match the current term structure. Black, Derman and Toy (1990) and Black and Karasinski (1991) (BK) develop lognormal diffusion models for the short rate that have the same no-arbitrage property. The model developed in this paper is a multifactor extension of the BK model, where the short rate follows a lognormal process with a stochastic central tendency. The lognormal models of Black, Derman and Toy (1990) and Black and Karasinski (1991) are perhaps closest to the model developed in this paper. These papers derive recombining, binomial lattices which match yield volatilities and cap-floor volatilities respectively. In a sense, our model can be viewed as a multi-factor extension of the Black and Karasinski model. In their model, the 
local (conditional) volatilities and the mean reversion of the short rate are given, in addition to the current term structure of zero-coupon bond prices. They build a recombining binomial tree of rates, consistent with this market information, using a technique whereby the length of the time period is changed to accommodate mean reversion and changing local volatilities. Unfortunately, as pointed out by Amin (1991), this "trick" only works, in general, for a one-factor model. In this paper, we therefore employ the changing probability technique of Nelson and Ramaswamy (1990), extended to multiple variables by HSS. We are thus able to generalise the Black-Karasinski model to two or more factors, while maintaining the recombining property.

In a no-arbitrage framework, HJM model the evolution of forward rates for various maturities. A similar approach has recently been used in the "market model" of Brace, Gatarek and Musiela, (1997) (BGM) and Miltersen, Sandmann and Sondermann (1997) (MSS). These papers, like this one, model the $L I B O R$ interest rate. Since spot rates and forward rates are closely related, our modelling approach can be compared to these papers. However, in contrast to these reduced-form models where the behaviour of forward rates is exogenous, our model is a structural-type model, where only the behaviour of the short $(L I B O R)$ rate and the premia of the first two futures rates are exogenous. Although it is possible to develop multifactor-forward rate models in the HJM framework, these often require restrictive assumptions to guarantee the Markov property, and the use of MonteCarlo simulation. The advantage of our methodology is that it is implementable in seconds, for general volatility structure assumptions. In some senses, forward-rate models can be regarded also as spot-rate models. However, except in the case of Gaussian interest rates, the relationship between the forward-rate process and the spot-rate process is complex. We directly build a no-arbitrage, multifactor spot rate model which has the Markov property. The model is then directly applied to the valuation of American-style and Bermudan-style interest rate derivatives.

A related paper is Heath (1998), who starts with the term structure of futures rates and then builds a no-arbitrage process for the term structure of futures rates. Having generated futures rates at each point in time, Heath proposes using a convexity adjustment to derive forward rates and bond prices. This methodology potentially avoids the non-Markov property characteristic of many forward-rate models. We also use futures $L I B O R$ and in particular we use the property that the futures $L I B O R$ is the expectation of the future spot rate under the risk-neutral measure. This enables us to calibrate our spot-rate model easily to the current term structure of futures prices.

A number of authors, including Hull and White (1994), Jegedeesh and Penachi, Balduzzi, Das and Foresi (1998) and Stapleton and Subrahmanyam (2001), have developed two-factor term-structure models where the second factor is a shock to the conditional mean of the 
spot rate. ${ }^{5}$ Hull and White propose a general class of two-factor models where the short rate mean reverts to a deterministic mean, although they only implement certain special cases of the class, where the term structure of volatility is restricted. Our incremental contribution is to implement a multi-factor model of the Hull-White type, but with a general volatility stucture, in a lognormal setting.

One recent paper that deals with the pricing of American-style and Bermudan-style swaptions is by Longstaff, Santa-Clara and Schwartz (2001). Their paper emphasizes the importance of including multiple factors in a pricing model for these claims. Our results support their conclusion. While our implementation only allows for two or three factors, we are able to price the contingent claims in a much faster, more efficient way, without resorting to the use of Monte-Carlo simulation. ${ }^{6}$ The current state-of-the-art on the pricing of American-style and Bermudan-style swaptions in the LIBOR market model is summarised in Andersen (2000). Various approximations have been proposed to circumvent the nonMarkov nature of the short-rate process. Andersen compares a number of methods and suggests the computation of a lower bound for the price of a Bermudan-style swaption based on a restricted factor model assumption, used for the purpose of taking the early exercise decision. Andersen and Andreasen (2001) test whether the number of factors has a significant impact on Bermudan-style swaption prices when the model is calibrated to the price of European-style swaptions. They find very small effects, both in the case of the Hull and White (1994) Gaussian model and in the case of the LIBOR market model.

Our paper is also related to two recent contributions of Rebonato (1999) and Sidenius (2000). These papers discuss methods of calibrating multi-factor LIBOR market models to both the cap implied volatilities and the prices of European-style swaptions. Our approach provides an alternative calibration methodology. The difference in the case of spot rate models, is that the correlation of the forward rates in the term structure is determined endogenously in these models. In the forward rate models, the calibration is to the pricing of interest rate options and an exogenously given correlation matrix.

\footnotetext{
${ }^{5}$ In a recent paper, Dai and Singleton (2000) explore the properties of affine term structure models, within which broad category they consider a class of models with a 'stochastic central tendency' such as that of Balduzzi, Das and Foresi (1998). The models proposed by Hull and White (1994) and Stapleton and Subrahmanyam (2001) are similar.

${ }^{6}$ Monte-Carlo simulation is an alternative approach to the problem of valuing options in multi-factor models. Both methods (recombining trees and Monte-carlo simulation) create their own inaccuracies due to their different ways of approximating the true distribution.
} 


\section{The Multi-Factor Model}

In this section, we describe our multi-factor model and investigate the implications of the no-arbitrage conditions for the model. We first discuss briefly the general approach in the lemmas and propositions that follow. Since our approach involves the calibration of the model using observable futures rates, we first establish the linkage between the spot and futures rates. The key to developing such a link is the observation that in an arbitragefree economy, futures prices are the expectation, under the risk-neutral measure, of the future spot prices. The other relationship we use is the expression for the mean of the spot interest rate process, based on the assumption of lognormality of the spot interest rate. These restrictions allow us to re-formulate the spot rate process in terms of futures rates. Having specified the spot-rate process, we then derive the process for the one-period and two-period ahead futures rates, using similar methods.

The logic of the argument is as follows. First, we show, in Lemma 1, that the futures rate is the expectation, under the risk-neutral measure, of the future spot interest rate. Since the spot rate is lognormally distributed, the futures rate can be related to the mean and variance of the $(\log )$ spot interest rate. Second, in Lemma 2, the spot interest rate process is expressed in terms of observable parameters by taking the expectation and substituting for the futures rate expressed as the mean of the spot interest rate. Third, in Lemma 3 , a crosssectional relation is derived between futures and spot rates. These results are combined in Proposition 1 with the requirement that forward bond prices are the expectation, under the risk-neutral measure of the future bond prices. Proposition 1 summarises the no-arbitrage requirements of the model.

\subsection{No-arbitrage properties of the model}

As several authors have noted, one way of introducing a second factor into a spot-rate model of the term structure is to assume that the conditional mean of the spot short-term interest rate is stochastic. Further factors may be added by then assuming that the conditional mean of the second and subsequent factors are also modelled with stochastic conditional means. ${ }^{7}$ In this paper, we take a similar approach. We assume that the logarithm of the short-term interest rate follows a discrete process with a stochastic conditional mean. In order to avoid complexity of notation, we present the model with three factors. We also consider a restricted two-factor version of the model which is more practical from an

\footnotetext{
${ }^{7}$ See, for example, Hull and White (1994), Balduzzi, Das and Foresi (1998), and Jegadeesh and Pennacchi (1996), as well as the synthesis by Dai and Singleton (2000).
} 
implementation viewpoint and which will be used extensively in the section on calibration of the model.

We define the short-term, $m$-year interest rate, on a $L I B O R$ basis as $r_{t}=\left[\left(1 / B_{t, t+m}\right)-1\right] / m$, where $m$ is a fixed maturity of the short rate and $B_{t, t+m}$ is the price of a $m$-year, zerocoupon bond at time $t$. We then assume that, under the (daily) risk-neutral measure, this rate follows the process: ${ }^{8}$

$$
\ln \left(r_{t}\right)-\ln \left(r_{t-1}\right)=\theta_{r_{t}}-b \ln \left(r_{t-1}\right)+\ln \left(\pi_{t-1}\right)+\varepsilon_{t},
$$

where

$$
\ln \left(\pi_{t}\right)-\ln \left(\pi_{t-1}\right)=\theta_{\pi_{t}}-c \ln \left(\pi_{t-1}\right)+\ln \left(z_{t}\right)+\nu_{t}
$$

and

$$
\ln \left(z_{t}\right)-\ln \left(z_{t-1}\right)=\theta_{z_{t}}-d \ln \left(z_{t-1}\right)+\eta_{t}
$$

and $\varepsilon_{t}, \nu_{t}$, and $\eta_{t}$ are possibly correlated, normal, random variables. $\pi$ is a shock to the conditional mean of the short-rate process, $z_{t}$ is a further shock to the mean of the $\pi_{t}$ process, $\theta_{r_{t}}, \theta_{\pi_{t}}$ and $\theta_{z_{t}}$ are time-dependent constants. $b, c$ and $d$ are the mean reversion coefficients of $r$ and $\pi$ and $z$ respectively. The mean and the unconditional standard deviation of the logarithm of the factors, $r_{t}, \pi_{t}$ and $z_{t}$ are $\mu_{r_{t}}, \sigma_{r_{t}}, \mu_{\pi_{t}}, \sigma_{\pi_{t}}$, and $\mu_{z_{t}}, \sigma_{z_{t}}$ respectively. We assume that the trading interval is one day, and that the LIBOR follows the process in (1) under the daily (rather than the continuous) risk-neutral measure. From here on, we refer to this "daily" risk-neutral measure as simply the risk-neutral measure. We also assume, without loss of generality, that $E\left(\pi_{t}\right)=1$ and $E\left(z_{t}\right)=1$, where the expectation is again taken under the risk-neutral measure. ${ }^{9}$

\footnotetext{
as follows:

$$
\begin{aligned}
\ln \left(r_{t}\right)-\ln \left(r_{t-1}\right) & =\theta_{r_{t}}-b_{0} \ln \left(r_{t-1}\right)+\ln \left(y_{1, t-1}\right)+\varepsilon_{0, t}, \\
\ln \left(y_{1, t}\right)-\ln \left(y_{1, t-1}\right) & =\theta_{y_{1, t}}-b_{1} \ln \left(y_{1, t-1}\right)+\ln \left(y_{2, t}\right)+\varepsilon_{1, t}, \\
\ldots & =\ldots \\
\ln \left(y_{n, t}\right)-\ln \left(y_{n, t-1}\right) & =\theta_{y_{n, t}}-b_{n} \ln \left(y_{n, t-1}\right)+\varepsilon_{n, t-1}
\end{aligned}
$$
}

${ }^{8}$ The multi-factor version of the model, with slightly changed notation to accommodate $n+1$ factors, is

The conditional mean of each factor is stochastic, and is driven by the subsequent factor in an embedded fashion.

${ }^{9}$ Note that the assumed process in equation (1) is the discrete form of the process

$$
d \ln (r)=\left[\theta_{r_{t}}-b \ln (r)+\ln (\pi)\right] d t+\sigma_{r}(t) d z_{1}
$$


The model in equation (1) is attractive because the second and third factors $\pi$ and $z$ are closely related to the futures rate, which is observable. In fact, as we shall show in Appendix A, the futures $L I B O R$ is the expectation of $r_{t}$ under the risk-neutral measure. Hence, the model lends itself to calibration given market inputs. To see this, we first derive some of the implications of the process assumed in equation (1), in a no-arbitrage economy.

We now state and prove a result that is central to the paper. The result is not new, since a similar result is derived by Sundaresan (1991), and used by MSS (1997) and BGM (1997). However, since it is crucial to the model developed in this paper, we include the proof in Appendix A. The lemma states that, given the definition of the LIBOR futures contract, the futures $L I B O R$ is the expected value of the spot rate, under the risk-neutral measure.

Lemma 1 (Futures LIBOR) In a no-arbitrage economy, the time-t futures LIBOR, for delivery at $T$, is the expected value, under the risk-neutral measure, of the time-T spot LIBOR, i.e.

$$
f_{t, T}=E_{t}\left(r_{T}\right)
$$

Also, if $r_{T}$ is lognormally distributed under the risk-neutral measure, then:

$$
\ln \left(f_{t, T}\right)=E_{t}\left[\ln \left(r_{T}\right)\right]+\frac{\operatorname{var}_{t}\left[\ln \left(r_{T}\right)\right]}{2},
$$

where the operator "var" refers to the variance under the risk-neutral measure.

$\underline{\text { Proof }}$

See Appendix A.

Lemma 1 allows us to substitute the futures rate directly for the expected value of the $L I B O R$ in the process assumed for the spot rate. In particular, the futures rate has a zero

\footnotetext{
where

$$
d \ln (\pi)=\left[\theta_{\pi_{t}}-c \ln (\pi)\right] d t+\sigma_{\pi}(t) d z_{2}
$$

and

$$
d \ln (z)=\left[\theta_{z_{t}}-d \ln (z)\right] d t+\sigma_{z}(t) d z_{3}
$$

In the above equations, $d \ln (r)$ is the change in the logarithm of the short rate, and $\sigma_{r}(t)$ is the instantaneous volatility of the short rate. The second and third factors, $\pi$ and $z$, themselves follow a diffusion process with means $\theta_{\pi}, \theta_{z}$ mean reversions $c$ and $d$ and instantaneous volatilities $\sigma_{\pi}(t), \sigma_{z}(t)$, and $d z_{1}, d z_{2}$ and $d z_{3}$ are standard Brownian motions. If the short rate follows the process in equation (2), it is lognormal over any discrete time period. The model above, restricted to two factors, is one of the cases considered by Hull and White (1994). Note that the continuous-time process is defined under the continuous risk-neutral measure which is slightly different from the "daily" measure used in this paper.
} 
drift, under the risk-neutral measure. We now use this result to solve for the constant parameters in our interest rate process in (1), i.e., to determine the constants $\theta_{r_{t}}, \theta_{\pi_{t}}$, and $\theta_{z_{t}}$. We have:

Lemma 2 (Spot-LIBOR Process) Suppose that the short-term interest rate follows the process in equation (1), under the risk-neutral measure, in a no-arbitrage economy. Then, since $f_{0, t}=E_{0}\left(r_{t}\right), \forall t$, the short rate process can be specified as

$$
\ln \left(r_{t}\right)-\ln \left(f_{0, t}\right)=\alpha_{r_{t}}+\left[\ln \left(r_{t-1}\right)-\ln \left(f_{0, t-1}\right)\right](1-b)+\ln \left(\pi_{t-1}\right)+\varepsilon_{t}
$$

where

$$
\ln \left(\pi_{t}\right)=\alpha_{\pi_{t}}+\ln \left(\pi_{t-1}\right)(1-c)+\ln \left(z_{t-1}\right)+\nu_{t},
$$

and

$$
\ln \left(z_{t}\right)=\alpha_{z_{t}}+\ln \left(z_{t-1}\right)(1-d)+\eta_{t}
$$

with

$$
\alpha_{r_{t}}=\frac{-\sigma_{r_{t}}^{2}}{2}+(1-b) \frac{\sigma_{r_{t-1}}^{2}}{2}+\frac{\sigma_{\pi_{t-1}}^{2}}{2}
$$

and

$$
\begin{gathered}
\alpha_{\pi_{t}}=\frac{-\sigma_{\pi_{t}}^{2}}{2}+(1-c) \frac{\sigma_{\pi_{t-1}}^{2}}{2}+\frac{\sigma_{z_{t-1}}^{2}}{2}, \\
\alpha_{z_{t}}=\frac{-\sigma_{z_{t}}^{2}}{2}+(1-d) \frac{\sigma_{z_{t-1}}^{2}}{2}
\end{gathered}
$$

$\underline{\text { Proof }}$

See Appendix B.

The result in Lemma 2 is important for the implementation of the model developed in this paper, since it defines the parameters of the three-factor interest rate process in terms of potentially observable quantities. The process for the $L I B O R$ depends upon the current futures rates and the volatilities of the $L I B O R$ and of the premium factors. Lemma 2 implies that if the no-arbitrage condition is to be satisfied, the drift of the spot rate process has to reflect the futures $L I B O R$ at time 0 and the volatilities. This is analogous to the no-arbitrage requirement in the HJM model, where the absence of arbitrage implies that the drift of the forward rate depends on the volatility of the forward rates. In our spot rate lognormal model, the volatilities of the spot rate and of the premium factor play a similar role. 
However, the condition used in Lemma 2, that $E_{0}\left(r_{t}\right)=f_{0, t}$, is necessary, but not sufficient, for "no-arbitrage" in our spot-futures model. The no-arbitrage requirement is much stronger. From Lemma 1, no-arbitrage requires that the futures LIBOR equals the expected spot rate at each date and in each state. We then have the following:

Lemma 3 (Futures-LIBOR Process) Given that the conditions of Lemma 2 are satisfied, the no-arbitrage condition implies

$$
\ln \left(f_{t, t+1}\right)-\ln \left(f_{0, t+1}\right)=\alpha_{f_{t+1}}+\left[\ln \left(r_{t}\right)-\ln \left(f_{0, t}\right)\right](1-b)+\ln \left(\pi_{t}\right)
$$

where

$$
\alpha_{f_{t+1}}=\alpha_{r_{t+1}}+\operatorname{var}_{t}\left[\ln \left(r_{t+1}\right)\right] / 2
$$

and

$$
\begin{aligned}
\ln \left(f_{t, t+2}\right)-\ln \left(f_{0, t+2}\right) & =\alpha_{f_{t+2}}+\left[\ln \left(r_{t}\right)-\ln \left(f_{0, t}\right)\right](1-b)^{2} \\
& +\ln \left(\pi_{t}\right)[(1-b)+(1-c)]+\ln \left(z_{t}\right)
\end{aligned}
$$

where

$$
\alpha_{f_{t+2}}=\alpha_{r_{t+2}}+(1-b) \alpha_{r_{t+1}}+\alpha_{\pi_{t+1}}+\operatorname{var}_{t}\left[\ln \left(r_{t+2}\right)\right] / 2
$$

$\underline{\text { Proof }}$

See Appendix C.

Lemma 3 shows that, in a no-arbitrage economy where the spot rate follows (3), the first futures contract has a rate that follows a two-factor process. The futures rate moves with changes in the spot rate, and in response to the premium factor, $\pi$. The futures rate is also affected by the degree of mean reversion in the short rate process. We can interpret the volatility of the premium factor as the part of the volatility of the first futures rate that is not explained by the spot rate. ${ }^{10}$

\footnotetext{
${ }^{10}$ It is natural to concentrate on the first futures rate, i.e., the futures for delivery at time $t+1$, since in our spot-rate model, the first futures rate is the expected value of the subsequent spot rate, $r_{t+1}$. However, it is possible to solve the time-series model for the $k$ th futures rate, to obtain:

$$
\ln \left(f_{t, t+k}\right)-\ln \left(f_{0, t+k}\right)=\alpha_{f_{t+k}}+\left[\ln \left(r_{t}\right)-\ln \left(f_{0, t}\right)\right](1-b)^{k}+V_{t} A_{t, k}
$$

where $V_{t}$ is a weighted sum of the innovations in the premium factor, and $A_{t, k}$ is a constant. Hence the $k$ th futures $L I B O R$ also follows a two-factor process similar to that followed by the first futures $L I B O R$.
} 
So far, we have concentrated on the implications of the no-arbitrage condition for the spotrate process and for futures rates. However, any term-structure model must also satisfy the condition that, under the risk-neutral measure, forward bond prices must equal the expected values of the subsequent period's bond price. This condition is therefore included in the following proposition that summarises the no-arbitrage conditions of our model.

Proposition 1 (No-Arbitrage Properties of the Model) Suppose that the LIBOR rate, $r_{t}$ follows the process:

$$
\ln \left(r_{t}\right)-\ln \left(r_{t-1}\right)=\theta_{r_{t}}-b \ln \left(r_{t-1}\right)+\ln \left(\pi_{t-1}\right)+\varepsilon_{t},
$$

where

$$
\ln \left(\pi_{t}\right)-\ln \left(\pi_{t-1}\right)=\theta_{\pi_{t}}-c \ln \left(\pi_{t-1}\right)+\nu_{t},
$$

and

$$
\ln \left(z_{t}\right)-\ln \left(z_{t-1}\right)=\theta_{z_{t}}-d \ln \left(z_{t-1}\right)+\eta_{t}
$$

under the risk-neutral measure, with $E\left(\pi_{t}\right)=1$ and $E\left(z_{t}\right)=1, \forall t$, and $\varepsilon_{t}, \nu_{t}$, and $\eta_{t}$ are independently distributed, normal variables. Then, if the model is arbitrage free:

1. the spot-LIBOR process can be written as:

$$
\ln \left(r_{t}\right)-\ln \left(f_{0, t}\right)=\alpha_{r_{t}}+\left[\ln \left(r_{t-1}\right)-\ln \left(f_{0, t-1}\right)\right](1-b)+\ln \left(\pi_{t-1}\right)+\varepsilon_{t},
$$

2. the process for the 1-period futures-LIBOR can be written as:

$$
\ln \left(f_{t, t+1}\right)-\ln \left(f_{0, t+1}\right)=\alpha_{f_{t+1}}+\left[\ln \left(r_{t}\right)-\ln \left(f_{0, t}\right)\right](1-b)+\ln \left(\pi_{t}\right),
$$

3. the process for the 2-period futures-LIBOR can be written as:

$$
\begin{aligned}
\ln \left(f_{t, t+2}\right)-\ln \left(f_{0, t+2}\right) & =\alpha_{f_{t+2}}+\left[\ln \left(r_{t}\right)-\ln \left(f_{0, t}\right)\right](1-b)^{2} \\
& +\ln \left(\pi_{t}\right)[(1-b)+(1-c)]+\ln \left(z_{t}\right)
\end{aligned}
$$

4. zero-coupon bond prices are given by the relation:

$$
B_{s, t}=B_{s, s+1} E_{s}\left(B_{s+1, t}\right), 0 \leq s<t \leq T .
$$


$\underline{\text { Proof }}$

Parts 1, 2 and 3 of the proposition follow from Lemmas 2, 3. As shown by Pliska (1997), Part 4 is a requirement of any no-arbitrage model.

Proposition 1 summarises the conditions that have to be met for the spot-futures model to be arbitrage-free. Also, as noted above, the further implication of Lemma 1, is that the futures rate is a martingale, under the risk-neutral measure. Hence, we can easily calibrate the model to the given term structure of futures rates, and thereby guarantee that the no-arbitrage property holds.

Finally, for completeness, we should note that the process followed by the spot and futures rates in this model can be written in difference form:-

Corollary 1 (The Multi-Variate Spot-Futures Process) The multi-variate process for the spot-LIBOR and the one-period and two-period ahead futures-LIBOR can be written as:

$$
\begin{aligned}
\Delta \ln \left(r_{t}\right) & =\alpha_{r_{t}}^{\prime}-b \ln \left(r_{t-1}\right)+\ln \left(\pi_{t-1}\right)+\varepsilon_{t} \\
\Delta \ln \left(f_{t, t+1}\right) & =\alpha_{f_{t, 1}}^{\prime}+\left[\ln \left(r_{t}\right)-\ln \left(r_{t-1}\right)\right](1-b)+\ln \left(\pi_{t}\right)-\ln \left(\pi_{t-1}\right), \\
\Delta \ln \left(f_{t, t+2}\right) & =\alpha_{f_{t, 2}}^{\prime}+\left[\ln \left(r_{t}\right)-\ln \left(r_{t-1}\right)\right](1-b)^{2} \\
& +\left[\ln \left(\pi_{t}\right)-\ln \left(\pi_{t-1}\right)\right][(1-b)+(1-c)]+z_{t+1}-z_{t},
\end{aligned}
$$

for some constants $\alpha_{r_{t}}^{\prime}, \alpha_{f_{t, 1}}^{\prime}$ and $\alpha_{f_{t, 2}}^{\prime}$.

Proof

Write equation (3) for $r_{t+1}$ and for $r_{t}$ and subtract the second equation from the first. Then the first part of the corollary follows with

$$
\alpha_{r_{t}}^{\prime}=\alpha_{r_{t+1}}-\alpha_{r_{t}}-(1-b) \ln \left(f_{0, t}\right)+(1-b) \ln \left(f_{0, t-1}\right) .
$$

Similarly, write equation (4) for $f_{t+1, t+2}$ and for $f_{t, t+1}$ and subtract the second equation from the first. Similarly, taking the first differnce of the equation for the two-period ahead, the corollary follows.

The first part of the corollary shows that the spot rate follows a one dimensional meanreverting process. The second part shows that the 1-period futures rate follows a twodimensional process, depending partly on the change in the spot rate and partly on the change in the premium factor. The third part shows that the 2-period futures rate follows a three-dimensional process, depending partly on the change in the spot rate and partly on the change in the first and second premium factors. 


\subsection{Regression Properties of the Model}

The two-factor model of the term structure described above has the characteristic that the conditional mean of the short rate is stochastic, as does the Hull and White (1994) model. Since the futures rate directly depends on the conditional mean, there is an imperfect correlation between the short rate and the futures rate. In this section, we establish the regression properties of the model, using the covariances of the short rate and premium process. These properties are required as inputs for the construction of a binomial approximation model of the term structure. In the following proposition, we denote the covariance of the logarithm of the short rate and the premium factor as $\sigma_{r_{t}, \pi_{t}}$. The process assumed in Lemma 2 has the following properties:

Proposition 2 (Multiple Regression Properties) Assume that

$$
\ln \left(r_{t}\right)-\ln \left(f_{0, t}\right)=\alpha_{r_{t}}+\left[\ln \left(r_{t-1}\right)-\ln \left(f_{0, t-1}\right)\right](1-b)+\ln \left(\pi_{t-1}\right)+\varepsilon_{t}
$$

where

$$
\ln \left(\pi_{t}\right)=\alpha_{\pi_{t}}+\ln \left(\pi_{t-1}\right)(1-c)+\ln \left(z_{t-1}\right)+\nu_{t},
$$

and

$$
\ln \left(\pi_{t}\right)=\alpha_{z_{t}}+\ln \left(z_{t-1}\right)(1-d)+\eta_{t},
$$

with $E_{0}\left(\pi_{t}\right)=1$ and $E_{0}\left(z_{\pi_{t}}, \forall t\right.$.

Then,

1. the multiple regression

$$
\ln \left[\frac{r_{t}}{f_{0, t}}\right]=\alpha_{r_{t}}+\beta_{r_{t}} \ln \left[\frac{r_{t-1}}{f_{0, t-1}}\right]+\gamma_{r_{t}} \ln \left(\pi_{t-1}\right)+\varepsilon_{t}
$$

has coefficients

$$
\begin{gathered}
\alpha_{r_{t}}=\left(-\sigma_{r_{t}}^{2}+\beta_{r_{t}} \sigma_{r_{t-1}}^{2}+\gamma_{r_{t}} \sigma_{\pi_{t-1}}^{2}\right) / 2 \\
\beta_{r_{t}}=(1-b), \\
\gamma_{r_{t}}=1,
\end{gathered}
$$

2. the multiple regression

$$
\ln \left(\pi_{t}\right)=\alpha_{\pi_{t}}+\beta_{\pi_{t}} \ln \left(\pi_{t-1}\right)+\gamma_{\pi_{t}} \ln \left(z_{t-1}\right)+\nu_{t}
$$


has coefficients

$$
\begin{gathered}
\alpha_{\pi_{t}}=\left[-\sigma_{\pi_{t}}^{2}+\beta_{\pi_{t}} \sigma_{\pi_{t-1}}^{2}+\gamma_{\pi_{t}} \sigma_{z_{t-1}}^{2}\right] / 2 \\
\beta_{\pi_{t}}=(1-c), \\
\gamma_{\pi_{t}}=1,
\end{gathered}
$$

3. the regression

$$
\ln \left(z_{t}\right)=\alpha_{z_{t}}+\beta_{z_{t}} \ln \left(z_{t-1}\right)+\eta_{t}
$$

has coefficients

$$
\begin{gathered}
\alpha_{z_{t}}=\left[-\sigma_{z_{t}}^{2}+\beta_{z_{t}} \sigma_{z_{t-1}}^{2}\right] / 2, \\
\beta_{z_{t}}=(1-d),
\end{gathered}
$$

4. the conditional variances of $\ln \left(r_{t}\right)$ and $\ln \left(\pi_{t}\right)$ are given by

$$
\begin{aligned}
\operatorname{var}_{t-1}\left(\varepsilon_{t}\right)= & \sigma_{r_{t}}^{2}-(1-b)^{2} \sigma_{r_{t-1}}^{2}-\sigma_{\pi_{t-1}}^{2}-2(1-b) \sigma_{r_{t-1}, \pi_{t-1}}, \\
\operatorname{var}_{t-1}\left(\nu_{t}\right)= & \sigma_{\pi_{t}}^{2}-(1-c)^{2} \sigma_{\pi_{t-1}}^{2}-\sigma_{z_{t-1}}^{2}-2(1-c) \sigma_{z_{t-1}, \pi_{t-1}}, \\
& \operatorname{var}_{t-1}\left(\eta_{t}\right)=\sigma_{z_{t}}^{2}-(1-d)^{2} \sigma_{z_{t-1}}^{2}
\end{aligned}
$$

where $\sigma_{r, \pi}$ denotes the annualized covariance of the logarithms of the short rate and the premium factor.

$\underline{\text { Proof }}$

See Appendix D.

Note that we require the multiple regression coefficients in order to build the binomial approximation of the multi-variate process, using our modification of the method of Ho, Stapleton and Subrahmanyam (1995). From the proposition, the $\beta$ coefficients simply reflect the mean-reversion of the short rate, and the premium factors. The $\gamma$ coefficients are all unity, reflecting the one-to-one relationship between $\pi$, the futures premium factor and the expected spot rate and the relationship between $z_{t}$ and the expected value of the premium factor. The $\alpha$ coefficients reflect the drift of the lognormal distribution, which depends on the variances of the variables. Part 4 of the Proposition gives an expression for the conditional variance of the logarithm of the short rate, the first premium factor and the second premium factor. 


\subsection{An Economic Interpretation of the Factors}

In order to build the two-factor version of the model outlined above, we need the parameters of the premium process, as well as those for the short rate process itself. The result in Proposition 2, part 4 gives the relationship of the conditional volatility of the short rate to the unconditional volatilities of the short rate, the volatility of the first premium factor, and the mean reversion of the short rate. We assume that the unconditional volatilities of the short rate are given, for example, observable from caplet/floorlet volatilities, and that the mean reversion is also given. The premium process, $\pi_{t}$, on the other hand, determines the extent to which the first futures rate differs from the spot rate in the model. Note that it is the first futures rate that is relevant, since it is this futures rate that determines the expectation of the subsequent spot rate, in the model. Since the first premium factor is not directly observable, we need to be able to estimate the mean and volatility of the premium factor from the behavior of futures rates. In order to discuss this, we first establish the following general result:

Lemma 4 Assume that

$$
\ln \left(r_{t}\right)-\ln \left(f_{0, t}\right)=\alpha_{r_{t}}+\left[\ln \left(r_{t-1}\right)-\ln \left(f_{0, t-1}\right)\right](1-b)+\ln \left(\pi_{t-1}\right)+\varepsilon_{t}
$$

where

$$
\ln \left(\pi_{t}\right)-\mu_{\pi_{t}}=\ln \left(\pi_{t-1}-\mu_{\pi_{t-1}}\right)(1-c)+\ln \left(z_{t-1}\right)+\nu_{t},
$$

with $E_{0}\left(\pi_{t}\right)=1, \forall t$, then the conditional volatility of $\pi_{t}$ is given by

$$
\sigma_{\pi}(t)=\left[\sigma_{x}^{2}(t)-(1-b)^{2} \sigma_{r}^{2}(t)\right]^{\frac{1}{2}}
$$

where $x=E_{t}\left(r_{t+1}\right)$ and $\operatorname{var}_{t-1}\left[\ln \left(x_{t}\right)\right]=\sigma_{x}^{2}(t)$.

$\underline{\text { Proof }}$

See Appendix E.

Lemma 4 relates the volatility of the premium factor to the volatility of the conditional expectation of the short rate. To apply this in the current context, we first assume that the short rate follows the process assumed in the lemma under the risk-neutral process. We then use the fact that the unconditional expectation of the $t+1$ th rate is $f_{t, 1}=E_{t}\left(r_{t+1}\right)$, i.e., the first futures (or forward) rate is the expected value of the next period spot rate. This implication of no-arbitrage leads to 


$$
\ln f_{t, 1}=\mu_{r_{t+1}}+\left[\ln r_{t}-\mu_{r_{t}}\right](1-b)+\ln \pi_{t-1}(1-c)+\nu_{t}+\sigma_{r}^{2}(t) / 2
$$

It follows that the conditional logarithmic variance of the first futures rate is given by the relationship

$$
\sigma_{f}^{2}(t)=(1-b)^{2} \sigma_{r}^{2}(t)+\sigma_{\pi}^{2}(t)
$$

Hence, the volatility of the premium factor is potentially observable from the volatility of the first futures rate. This, in turn, could be estimated empirically or implied from the prices of options on the $L I B O R$ futures rate. A similar argument can be used to derive the volatility of the second premium factor from the second futures rate.

\section{The Multivariate-Binomial Approximation of the Process}

In order to implement the model with a binomial approximation, we need to construct a recombining lattice for the spot rate, $r_{t}$, and the futures rate, $f_{t, t+1}$. A number of methods have been suggested in the literature. For example, Hull and White (1994) use a trinomial tree, but they assume a special case of non-time-dependent volatility, which is not realistic, in general. Amin (1991) and Black and Karasinski (1991) redefine the time interval between points on the grid to cope with changing local volatility. However, as noted by Amin (1991), this technique only works in the univariate case, or when the volatility functions and mean reversions are the same for each variable. In his multivariate implementation, Amin (1991) assumes time-independent volatilities. Nelson and Ramaswamy (1990) use a transformation of the process and state-dependent probabilities, to approximate a univariate diffusion. In an extension to multivariate diffusions, and in the special case, relevant here, of lognormal diffusions, Ho, Stapleton and Subrahmanyam (1995) use the regression properties of the multivariate diffusion to compute the appropriate probabilities of up-moves on the multivariate binomial tree. This allows them to capture both the time series and cross-sectional properties of the process. In this section we use a modification of their methodology.

\subsection{The HSS approximation}

Here we describe our approach for the case of the two-factor implementation of the model. The method we use for building a bivariate-binomial lattice, representing a discrete approx- 
imation of the process in equation (2), is to construct two separate recombining binomial trees for the short-term interest rate and the futures-premium factors. The no-arbitrage property and the covariance characteristics of the model are then captured by choosing the conditional probabilities at each node of the tree. The recombining nature of the bivariate tree is illustrated in Figure 1 for a two-period example and in Figure 2 for a three-period example. As shown in the figures, there are two possible outcomes emanating from each node. However, since the tree is required to recombine, it does not result in an explosive state space.

We now outline our method for approximating the two-factor process interest rate process, described above. We use three types of inputs: first, the unconditional means of the shortterm rate, $E_{0}\left(r_{t}\right), t=1, \ldots, T$, second, the volatilities of $\varepsilon_{t}$, i.e., the conditional volatility of the short rate, given the previous short rate and the previous futures rate, denoted by $\sigma_{r}(t)$, and the conditional volatilities of the premium, denoted by $\sigma_{\pi}(t)$, and third, estimates of the mean reversion of the short rate, $b$, and the mean reversion, of the premium factor, $c$. The process in (3) is then approximated using an adaptation of the methodology described in Ho, Stapleton and Subrahmanyam (1995) (HSS). HSS show how to construct a multiperiod multivariate-binomial approximation to a joint-lognormal distribution of $M$ variables with a recombining binomial lattice. However, in the present case, we need to modify the procedure, allowing the expected value of the interest rate variable to depend upon the premium factor. That is, we need to model the two variables $r_{t}$ and $\pi_{t}$, where $r_{t}$ depends upon $\pi_{t-1}$. Furthermore, in the present context, we need to implement a multiperiod process for the evolution of the interest rate, whereas HSS only implement a two-period example of their method. In this section, these modifications and the resulting multiperiod algorithm are presented in detail.

We divide the total time period into $T$ periods of equal length of $m$ years, where $m$ is the maturity period, in years, of the short-term interest rate. Over each of the periods from $t$ to $t+1$, we denote the number of binomial time steps, termed the binomial density, by $n_{t}$. Note that, in the HSS method, $n_{t}$ can vary with $t$ allowing the binomial tree to have a finer density, if required for accurate pricing, over a specified period. This might be required, for example, if the option exercise price changes between two dates, increasing the likelihood of the option being exercised, or for pricing barrier options.

We use the following result, adapted from HSS:

Proposition 3 (Approximation of a Two-factor Lagged Diffusion Process) Suppose that $X_{t}, Y_{t}$ follows a joint lognormal process, where $E_{0}\left(X_{t}\right)=1, E_{0}\left(Y_{t}\right)=1 \forall t$, and where

$$
E_{t-1}\left(x_{t}\right)=a_{x}+b x_{t-1}+y_{t-1}
$$




$$
E_{t-1}\left(y_{t}\right)=a_{y}+c y_{t-1} .
$$

Let the conditional logarithmic standard deviation of $Z_{t}$ be $\sigma_{z}(t)$ for $Z=(X, Y)$. If $Z_{t}$ is approximated by a log-binomial distribution with binomial density $N_{t}=N_{t-1}+n_{t}$ and if the proportionate up and down movements, $u_{z_{t}}$ and $d_{z_{t}}$ are given by

$$
\begin{aligned}
d_{z_{t}} & =\frac{2}{1+\exp \left(2 \sigma_{z}(t) \sqrt{1 / n_{t}}\right)} \\
u_{z_{t}} & =2-d_{z_{t}}
\end{aligned}
$$

and the conditional probability of an up-move at node $r$ of the lattice is given by

$$
q_{z_{t}}=\frac{E_{t-1}\left(z_{t}\right)+\left(N_{t-1}-r\right) \ln \left(u_{z_{t}}\right)-\left(n_{t}+r\right) \ln \left(d_{z_{t}}\right)}{n_{t}\left[\ln \left(u_{z_{t}}\right)-\ln \left(d_{z_{t}}\right)\right]}
$$

then the unconditional mean and conditional volatility of the approximated process approach their true values, i.e., $\hat{E}_{0}\left(Z_{t}\right) \rightarrow 1$ and $\hat{\sigma}_{z_{t}} \rightarrow \sigma_{z_{t}}$ as $n \rightarrow \infty$.

Proof

If $E_{0}\left(Z_{t}\right)=1, \forall t$, then we obtain the result as a special case of $\operatorname{HSS}(1995)$, Theorem $1 . \square$

\subsection{Computing the nodal values}

In this section, we first describe how the vectors of the short-term rates and the premium factor are computed. We approximate the process for the short-term interest rate, $r_{t}$, with a binomial process, i.e., moves up or down from its expected value, by the multiplicative factors $d_{r_{t}}$ and $u_{r_{t}}$. Following HSS, equation (7), these are given by

$$
\begin{aligned}
& d_{r_{t}}=\frac{2}{1+\exp \left(2 \sigma_{r}(t) \sqrt{1 / n_{t}}\right)} \\
& u_{r_{t}}=2-d_{r_{t}} .
\end{aligned}
$$

We then build a separate tree of the futures premium factor $\pi$. The up-factors and downfactors in this case are given by 


$$
\begin{aligned}
& d_{\pi_{t}}=\frac{2}{1+\exp \left(2 \sigma_{\pi}(t) \sqrt{1 / n_{t}}\right)} \\
& u_{\pi_{t}}=2-d_{\pi_{t}} .
\end{aligned}
$$

At node $j$ at time $t$, the interest rates $r_{t}$ and premium factors $\pi_{t}$ are calculated from the equations

$$
\begin{aligned}
r_{t, j} & =u_{r_{t}}^{\left(N_{t}-j\right)} d_{r_{t}}^{j} E_{0}\left(r_{t}\right) \\
\pi_{t, j} & =u_{\pi_{t}}^{\left(N_{t}-j\right)} d_{\pi_{t}}^{j} \\
j & =0,1, \ldots, N_{t}
\end{aligned}
$$

where $N_{t}=\sum_{t} n_{t}$. In general, there are $N_{t}+1$ nodes, i.e., states of $r_{t}$ and $\pi_{t}$, since both binomial trees are recombining. Hence, there are $\left(N_{t}+1\right)^{2}$ states after $t$ time steps.

\subsection{Computing the conditional probabilities}

In general, as in Hull and White (1994), the covariance of the two approximated diffusions may be captured by varying the conditional probabilities in the binomial process. Since the trees of the rates and the futures premium are both recombining, the time-series properties of each variable must also be captured by adjusting the conditional probabilities of moving up or down the tree, as in HSS and in Nelson and Ramaswamy (1990). Since, increments in the premium variable are independent of $r_{t}$, this is the simplest variable to deal with. Using the results of Proposition 2, we compute the conditional probability using HSS, equation (10). In this case the probability of a up-move, given that $\pi_{t-1}$ is at node $j$, is

$$
q_{\pi_{t}}=\frac{\alpha_{\pi_{t}}+\beta_{\pi_{t}} \ln \pi_{t-1, j}-\left(N_{t-1}-j\right) \ln u_{\pi_{t}}-\left(j+n_{t}\right) \ln d_{\pi_{t}}}{n_{t}\left(\ln u_{\pi_{t}}-\ln d_{\pi_{t}}\right)}
$$

where

$$
\begin{gathered}
\beta_{\pi_{t}}=(1-c) \\
\alpha_{\pi_{t}}=\left(-\sigma_{\pi_{t}}^{2}+\beta_{\pi_{t}} \sigma_{\pi_{t-1}}^{2}\right) / 2
\end{gathered}
$$


and where $b$ is the coefficient of mean reversion of $\pi$, and $\sigma_{\pi_{t}}^{2}$ is the unconditional logarithmic variance of $\pi$ over the period $(0-t)$.

The key step in the computation is to fix the conditional probability of an up-movement in the rate $r_{t}$, given the outcome of $r_{t-1}$, the mean reversion of $r$, and the value of the premium factor $\pi_{t-1}$. In discussing the multiperiod, multi-factor case, HSS present the formula for the conditional probability when a variable $x_{2}$ depends upon $x_{1}$ and a contemporaneous variable, $y_{2}$. Again using the regression properties derived in Proposition 2, and adjusting HSS, equation (13) to the present case, we compute the probability

$$
q_{r_{t}}=\frac{\alpha_{r_{t}}+\beta_{r_{t}} \ln \left(r_{t-1, j} / E\left(r_{t-1}\right)\right)+\gamma_{r_{t}} \ln \pi_{t-1, j}-\left(N_{t-1}-j\right) \ln u_{r_{t}}-\left(j+n_{t}\right) \ln d_{r_{t}}}{n_{t}\left(\ln u_{r_{t}}-\ln d_{r_{t}}\right)}
$$

where

$$
\begin{gathered}
\beta_{r_{t}}=(1-b) \\
\gamma_{r_{t}}=1 \\
\alpha_{r_{t}}=\left[-\sigma_{r_{t}}^{2}+\beta_{r_{t}} \sigma_{r_{t-1}}^{2}+\gamma_{r_{t}} \sigma_{\pi_{t}}^{2}\right] / 2 .
\end{gathered}
$$

Then, by Proposition 3, the process converges to a process with the given mean and variance inputs.

\subsection{The multiperiod algorithm}

HSS(1995) provide the equations for the computation of the nodal values of the variables, and the associated conditional probabilities, in the case of two periods $t$ and $t+1$. Efficient implementation requires the following procedure for the building of the $T$ period tree. The method is based on forward induction. First, compute the tree for the case where $t=1$. This gives the nodal values of the variables and the conditional probabilities, for the first two periods. Then, treat the first two periods as one new period, but with a binomial density equal to the sum of the first two binomial densities. The computations are carried out for period three nodal values and conditional probabilities. Note that the equations for the up-movements and down-movements of the variables always require the conditional volatilities of the variables in order to compute the vectors of nodal values. The following steps are implemented:

1. Using equation (11), compute the $\left[n_{1} \times 1\right]$ dimensional vectors of the nodal outcomes of $r_{1}, \pi_{1}$ with inputs $\sigma_{r}(1), E\left(r_{1}\right), \sigma_{\pi}(1), E\left(\pi_{1}\right)$ and binomial density $n_{1}$. Also, compute 
the $\left[\left(n_{1}+n_{2}\right) \times 1\right]$ dimensional vectors $r_{2}, \pi_{2}$ using inputs $\sigma_{r}(2), E\left(r_{2}\right), \sigma_{\pi}(2), E\left(\pi_{2}\right)$ and binomial density $n_{2}$. Assume the probability of an up-move in $r_{1}$ is 0.5 and then compute the conditional probabilities $q_{\pi_{1}}$ using equation (12) with $t=1$. Then, compute the conditional probabilities $q_{r_{2}}, q_{\pi_{2}}$, using equations (12) and (13), with $t=2$.

2. Using equation (11), compute the $\left[\left(N_{2}+n_{3}\right) \times 1\right]$ dimensional vectors $r_{3}, \pi_{3}$ using inputs $\sigma_{r}(3), E\left(r_{3}\right), \sigma_{r}(3), E\left(r_{3}\right)$ and binomial density, $n_{3}$. Then, compute the conditional probabilities $q_{r_{3}}, q_{\pi_{3}}$ using equations (12) and (13) with $t=3$.

3. Continue the procedure until the final period $T$.

In implementing the above procedure, we first complete step 1 , using $t=1$ and $t=2$, and with the given binomial densities $n_{1}$ and $n_{2}$. To effect step 2, we then redefine the period from $t=0$ to $t=2$ as period 1 and the period 3 as period 2 and re-run the procedure with a binomial densities $n_{1}^{*}=n_{1}+n_{2}$ and $n_{2}^{*}=n_{3}$. This algorithm allows the multiperiod lattice to be built by repeated application of equations (11), (12) and (13).

\subsection{A summary of the approximation method}

We will summarize the methodology by using a two-period and a three-period example. Figure 1 shows the recombining nodes for the two-factor process in the two-period case. The interest rate goes up to $r_{1,0}$ or down to $r_{1,1}$ at $t=1$. The futures premium factor goes up to $\pi_{1,0}$ or down to $\pi_{1,1}$ at $t=1$, with probability $q_{\pi_{1}}$. In the second period, there are just three nodes of the interest rate tree, together with three possible premium factor values. There are nine possible states, and the probability of an $r_{2}$ value materialising is $q_{r_{2}}$. Note that this probability depends on the level of the premium factor and of the interest rate at time $t=1$. The recombining property of the lattice, which is crucial for its computability, is emphasised in Figure 2, where we show the process for the interest rate over periods $t=2$ and $t=3$. After two periods, there are three interest rate states and nine states representing all the possible combinations of the interest rate and premium factor. The interest rate then goes to four possible states at time $t=3$ and there are sixteen states representing all the possible combinations of rates and premium factor. Note that the probability of reaching an interest rate at $t=3$ depends on both the interest rate and the premium factor at $t=2$. These are the probabilities that allow the no-arbitrage property of the model to be fulfilled. In the model, the term structure at time $t$ is determined by the two factors, one representing the short rate and the premium factor. Thus, with a binomial density of $n=1$, there are $(t+1)^{2}$ term structures generated by the binomial approximation, at time $t$. 


\section{The Two-Factor Model: Examples of Inputs and Outputs}

This section documents the results from several numerical examples based on the two-factor term structure model described in previous sections. First, we show that a two-factor term structure model can be implemented in a speedy and efficient manner. then we present the output from running a forty-eight quarter model, including the pricing of European-style, Bermudan-style and American-style swaptions.

In the numerical examples that follow, we choose a period length of three months. This is convenient for two reasons. First, we can model three-month LIBOR and then compute the corresponding maturity bond prices up to a given horizon without the added complexity of overlapping periods. Also, it enables the computational time to be reduced compared to a daily time interval model. However, changing the time interval does introduce one approximation. Theoretically, we need to use futures prices from contracts that are markedto-market at the same periodicity as the time interval in the model; otherwise, lemma 1 does not strictly apply. However, only daily marked-to-market prices are widely available. In calibrating the three-month period model to market data, a convexity adjustment may be required to adjust futures prices from a daily to a quarterly marked-to-market basis. In practice, this adjustment is likely to be very small, especially compared with the problems of obtaining long-maturity futures prices. ${ }^{11}$

\subsection{Computing Time}

Apart from the accuracy of the model, the most important feature of the methodology for implementing a two-factor model proposed in this paper is the computation time. It goes without saying that with two stochastic factors rather than one, the computation time can easily increase dramatically. In Table 1, we illustrate the efficiency of our model by showing the time taken to compute the zero-coupon bond prices and option prices. With a binomial density of one, the 48-period model takes 4.8 seconds and the 72-period model takes 17.2 seconds. Doubling the number of periods increases the computer time by a factor of six. There is clearly a trade-off between the number of periods, the binomial density of each period, and the computation time for the model. This is illustrated by the second line in the table, showing the effect of using a binomial density of two. Again the computation time increases more than proportionately as the density increases. The time taken for the

\footnotetext{
${ }^{11}$ The difference between daily and three-monthly marked-to-market futures $L I B O R$ is probably less than one basis point. For long maturities, lack of liquid futures contracts means that we have to estimate forward rates and apply a convexity adjustment. In this case the convexity adjustment is far more significant. See Gupta and Subrahmanyam (2000), for empirical estimates.
} 
24-period model, when the binomial density is two, is roughly the same as that for the 48-period model with a density of one.

\section{Calibration of the Model to Cap and Swaption Volatilities}

There are several different ways in which the two-factor version of the model can be calibrated to market prices of interest-rate caplets and or swaptions. In this section we illustrate two alternative calibration methods, using actual market data from one particular day. In both methods, we assume that the model parameters are stable through time and that the local volatilities are constant. The two methods of calibration proceed by solving for the model parameters that best fit the observed data. They are:

1. A calibration of the two-factor version of the model, using only the observed caplet volatilities .

2. A calibration of the two-factor model using both the observed caplet volatilities and the European-style swaption volatilities quoted on the same day.

\subsection{Market Data Used in the Calibration Exercise}

The data on the prices of US\$ caplets were collected for the calender years 1999 and 2000. On almost all days, the data reveal a term-structure of volatility for at-the-money caplets that is hump-shaped. The alternative methods of calibration are illustrated using data from a particular day, July 18, 2000. For example, for the date chosen for the calibration, the caplet quotes, in terms of Black volatilities, were as shown in Table 2. The futures LIBOR rates on the same day are shown in Table 3 . The futures rates are derived by interpolation from $L I B O R$ prices on the same day. On that day, the futures curve was rising quite steeply, from around $7 \%$ to nearly $8 \%$. Using this information, we estimated the at-the-money caplet quotes, from 12-months to 84-months maturity. These are shown in the second column of Table 4 and reveal an inverted U-shaped curve. ${ }^{12}$

Swaptions (European-style options to enter swaps) are quoted for at-the-money contracts for various maturities and for annual underlying swap tenors. As an example, the swaption

\footnotetext{
${ }^{12}$ This is typical of the data. We repeated the exercise for a previous date: 24 , November, 1999 . The only difference on that date was that the volatility curve had a slightly higher peak, the curve rising from $13.1 \%$ to a peak of $18.6 \%$ before falling to $16.0 \%$
} 
price quote matrix for July 18, 2000 is reproduced as Table 5, for swap maturities of 1year up to 5-years. Note that the swaption volatilities show a hump-shape similar to the caplets, but the hump is somewhat less pronounced. Also, the swaption volatilities are generally lower than the corresponding caplet volatilities, perhaps due to the lack of perfect correlation between forward rates.

\subsection{Method 1: Calibration of the Two-Factor Model to Caplet Volatilities}

In the first method, we calibrate the model to the caplet volatilities by running the model with the given values of the four parameters; $\sigma_{r}, b, \sigma_{\pi}$, and $c$. These are the (constant) local volatility of the $L I B O R$, the mean reversion of $L I B O R$, the (constant) local volatility of the futures premium factor, and the mean reversion of the futures premium factor. We assume that we require the model to price the caplets accurately, when it is run for a large value of the binomial density, $n$. After testing for the convergence of caplet prices, we used Richardson extrapolation to predict the price of each caplet for $n \rightarrow \infty$, given the prices for $n=3$ and $n=4 .^{13}$ The parameters that best fit the caplet prices, quoted as Black volatilities, in Table 4, are as follows: $\sigma_{r}=0.099, b=1.7, \sigma_{\pi}=0.025$, and $c=0.13$. All parameters are annualized. ${ }^{14}$ The caplet prices produced by the two-factor model are shown in the third column of Table 4. The root mean square error (RMSE) from comparing the model values to market quotes is $0.21 \% .^{15}$

An interesting question to answer is the following. Given the calibration of the two-factor model to the market caplet prices, how well does the model perform in predicting the market swaption volatility matrix? In a sense, this is a test (although only carried out with one day's data) of the cross-sectional performance of the model. The swaptions with maturities from one to five years, on swaps with a tenor of one to five years, were priced using the two-factor model, and then quoted as Black volatilities. The results are shown in Table 6 . The results show clearly that the model, when calibrated to the caplet prices, overprices the European-style swaptions. ${ }^{16}$ The RMSE for the swaptions is $1.67 \%$. The overpricing is

\footnotetext{
${ }^{13}$ Richardson extrapolation can be used because the caplet prices converge uniformly from below (as shown by tests using $n=1$ to $n=8$. For a discussion of Richardson approximation, see Schmidt (1968)

${ }^{14} \mathrm{On}$ a quarterly basis therefore the mean reversion of LIBOR is 0.425 and the mean reversion of the second factor is 0.0325 .

${ }^{15}$ In a recent empirical study of two-factor term-structure models Dai and Singleton (2000) note that evidence suggests that the short rate mean reverts relatively rapidly to a "stochastic tendency" that itself mean reverts slowly to a long run value. This is consistent with the results of the calibration reported here. It can be shown that the model used here is a lognormal version of the "stochastic tendency" class of models.

${ }^{16}$ This is consistent with the results of Longstaff, Santa-Clara and Schwartz (2000) who find that a "string" model calibrated to swaption prices underprices interest-rate caps.
} 
greatest for the short-maturity options and for the options on relatively short-tenor swaps. The model is relatively accurate when pricing the long-maturity options on long-tenor swaps. For example, for those swaptions where the sum of the option maturity and tenor exceed seven years, the average misspricing is $0.44 \%$. The only swaptions that are underpriced by the two-factor model are the five-year maturity options on the four-year and five-year tenor swaps.

\subsection{The Effect of Adding a Third Factor}

We now calibrate the three-factor version of the model, in order to check whether the mispricing of the swaptions can be reduced by adding a third factor. After a series of experiments it became apparent that the optimal way to introduce the third factor is to assume a white noise process for this factor. Hence, we tried various values for the volatility $\sigma_{z}$, with a mean reversion of $100 \%$. In this extreme case, the caplet volatilities are unaffected by adding a white noise third factor. Hence, the calibration process is straightforward. The third factor, in effect, partially explains the second factor. We are thus able to vary the $z$ volatility, to best fit the swaption volatility matrix, while leaving the caplet volatilities unaffected.

The model parameters which minimise the swaption volatility RMSE, while keeping the caplet RMSE at its previous minimised value of $0.21 \%$, are $\sigma_{r}=0.099, b=1.7, \sigma_{\pi}=0.092$, $c=0.13, \sigma_{z}=0.07$, and $d=4$. The resulting swaption volatilities are shown in Table 7. Note that the introduction of the third factor reduces all the swaption volatilities. However, the effect is smaller for the swaptions with longer underlying maturities. The overall RMSE is reduced to $0.98 \%$. Also, on average the errors are approximately zero. However some systematic biases remain. In particular, the short maturity (12-month) swaptions are overpriced and the long maturity (60-month) swaptions are now underpriced by the model. Also, the increased accuracy for the swaptions comes at the expence of a considerable increase in computing time.

\subsection{The Pricing of Bermudan-Style Swaptions and Yield-Spread Options}

We now use the calibrated model to price two contracts: a Bermudan-style swaption and a Bermudan-style yield-spread option. The results, along with the prices of comparable European-style options are shown in Tables 8 and 9 respectively. The Bermudan-style swaption is a payer swaption on a swap with a final maturity of six years, and a strike rate of $6.5 \%, 7.0 \%$, or $7.5 \%$. The two-factor model, with a binomial density of $n=2$, gives a 
price of 414 basis points for the Bermudan-style swaption, exerciseable annually at $6.5 \%$. This compares with the price of 365 basis points for the comparable one-year European-style option on a five-year swap. However, these prices are somewhat understated for the twofactor model, as shown by the results in the second panel of Table 8, where the model values are given for $n=3$. The Bermudan swaption price of 421 indicates that a more accurate estimate using Richardson extrapolation is 428 basis points. ${ }^{17}$ We can now investigate the effect of adding a third factor in the model. As noted above, the third factor reduces European-style swaption prices, due to the de-correlation effect on the forward rates. The Bermudan prices are also lower. Using Richardson extrapolation, the best estimate of the Bermudan from the three-factor model is 385 basis points, 43 points less than the two-factor price. The five-year European option is worth 342 basis points, 35 points less than in the two-factor case. ${ }^{18}$

A second application of the model is to price options on the difference between two interest rates. These options are generally known as yield-spread options. In Table 9, we show the results of using our model to price options on the spread between the four-year and the one-year rate. The options are either European-style options with a maturity of five years or Bermudan-style options, exerciseable annually up to a final maturity of five years. The options have a strike price of either $0 \%$ or $0.5 \%$. The results in Table 9 show that the valuation is sensitive to both the binomial density $(n)$ and the number of factors. To take account of the binomial density effect we again use Richardson extrapolation. The twofactor model then gives a European price of 20.0 basis points and the three-factor model gives a lower price of 17.6 basis points. Yield spreads are particularly sensitive to the exact model of the interest rate process, so it is not surprising that prices of options on spreads are highly sensitive to the number of factors. The Bermudan-style yield-spread options are somewhat less sensitive to the number of factors in the model. Again, using the Richardson extrapolation prices, the $0 \%$ strike option has a price of 30.8 basis points in the two-factor model and 30.0 in the three-factor model. As expected, the out of the money (strike rate

${ }^{17}$ The Richardson extrapolation estimate is given by

$$
\begin{aligned}
V(n=\infty) & =V(n=2)+2[V(n=3)-V(n=2)] \\
& =421+2(7) \\
& =435
\end{aligned}
$$

${ }^{18}$ The Richardson extrapolation estimates are given by

$$
\begin{aligned}
& =389+2(-2)=385 \\
\text { and } & =356+2(-7)=342
\end{aligned}
$$


of $0.50 \%$ ) Bermudan is somewhat more sensitive to the number of factors.

\subsection{Method 2: Calibration of the Two-Factor Model to Caplet and Swap- tion Volatilities}

The calibration of the two-factor model to caplet volatilities above leads to an overvaluation of swaptions. In this section we calibrate the model to both caplets and swaption volatilities, with differing weights placed on the two data sets. In this calibration we use a two-factor model with a binomial density of $n=2$. We price the swaptions, observe the errors compared to the market swaption quotes, and then change the four parameters of the model to minimise the RMSE of the swaption and the caplet prices, with a weight of 0.75 was placed on the caplet RMSE and 0.25 on the swaption RMSE. The result of this procedure is the choice of $\sigma_{r}=0.098, b=2.65, \sigma_{\pi}=0.1225$, and $c=0.087$.

The caplet volatilities produced by this parameterisation of the model are shown in column 4 of Table 4. The RMSE for the caplets is $0.87 \%$, compared to $0.21 \%$ in the previous calibration to the caplets alone. The short to medium term maturity caplets are overpriced by this model. The swaption volatility matrix which this resulted in is shown in Table 10. The RMSE for the swaption volatilities is $0.65 \%$. The model obviously fits the swaptions better than in the previous calibration. However, the short-maturity swaptions are still overpriced by the model. The long-maturity $(60$ month $)$ swaptions are slightly underpriced by the model. However, all the swaptions with maturities over 48 months or more are priced within at most $0.50 \%$ of the market volatility.

We now re-price a Bermudan-style swaption on a six-year underlying swap using the twofactor model calibrated to the European swaption prices. As before, the Bermudan-style option has the feature that it is exercisable at the end of each year up to the option maturity in year five. The European-style swaptions are one-year options on one-year to five-year swaps. Note that the model uses twenty-four quarterly time periods, to cover the six-year life of the underlying coupon bond. Table 11 shows the values of European and Bermudan swaptions at differing depths-in-the-money. Table 11 shows that the Bermudan option is worth considerably more than the European one-year option on a five-year swap. Comparing the results in Table 11 with those in the first panel of Table 8 shows that calibration of the model to the swaptions and the caplets (Table 11) has only a marginal effect on the Bermudan-style swaption price, in fact only 7 basis points. This suggests that the two-factor model calibrated to caplets alone does a fairly good job of pricing the Bermudan-style claim. 


\section{Conclusions}

Spot-rate models of the term structure can be regarded as structural models, while forward rate models are reduced form models. While forward-rate models of the general HJM type are useful for the pricing of European-style claims on interest rates, these models encounter some problems both with the valuation of European-style swaptions, and with the pricing of Bermudan-style claims. In order to price European-style swaptions, a forward rate model requires the exogenous specification of the forward rate correlation matrix. In contrast, in the structural term structure models in the literature, the correlation of forward rates is implicit in the models themselves. Further, the valuation of Bermudan-style claims within a forward rate model requires the derivation of a process for the spot rates which is, in general, non-Markov and complex. While various methods have been suggested to overcome these problems, it appears that a more natural approach is to directly model the spot rate process.

In this paper we have presented a model of the term structure of interest rates which can be regarded as a multi-factor extension of the Black-Karasinski lognormal-rate model. As in that model, we assume that the short-term LIBOR follows a lognormal process. However, in our model, a second factor determines the stochastic conditional mean of the short rate. The third and subsequent factors determine the conditional mean of the previous factor in a nested fashion. This model lends itself to an intuitively appealing interpretation in terms of futures rates. The first factor is identified with the spot rate, the second factor with the premium of the first futures rate over the spot, and the third factor with the premium of the second futures rate over first futures rate. We have shown that, by calibrating to the current term structure of futures rates, the model can be made arbitrage-free in the sense of Ho and Lee (1986) and Pliska (1997).

The model has been implemented by using the multivariate-binomial tree approach of Ho, Stapleton and Subrahmanyam (1995), using a recombining tree, in multiple dimensions, which has a non-exploding number of nodes. The no-arbitrage property, that the expected value of the spot rate under the risk-neutral measure is the one-period-ahead futures rate, is captured by adjusting the conditional probabilities of moving up the tree. Richardson extrapolation is used to increase the accuracy of the option prices.

In an illustrative example, the two-factor version of the model was calibrated both to caplet and to a combination of caplet and swaption price data. The model calibrated to caplet prices, under the assumption of constant local volatilities and mean reversion coefficients, over-priced the swaptions. This is consistent with previous results reported in Rebonato and Cooper (1995) and Longstaff, Santa-Clara and Schwartz (2000). Our limited evidence suggests that a two-factor model with uncorrelated errors, calibrated to caplet prices, cannot 
correctly price European-style swaptions. However, introducing a third, white noise, factor considerably reduces the pricing errors.

We then applied the two-factor model to the valuation of Bermudan-style payer swaptions. The model was also applied to the valuation of a variety of exotic options on interest rates, such as options on yield spreads. The model is particularly well suited to the valuation of Bermudan-style claims, since it directly models the spot-rate process. This is in contrast to many forward rate models where the implicit spot-rate process is non-Markov. As a consequence, the exercise decision is often difficult to capture in those models.

Our finding that the two-factor model, with uncorrelated errors, fails to accurately fit both the caplet and swaption prices, suggests two possible extensions of our two-factor model. First, as suggested here, we could add a third factor, which itself explains the stochastic mean of the second factor. Preliminary results show that the swaption pricing errors can be considerably reduced in such a model, especially when the mean reversion of the second factor is low. A further avenue for future research is to explore the effects of correlated errors. Such a model could help to capture the relatively low correlation of the spot and forward rates, which could again explain the swaption prices.

A possible further extension of the model would apply to the pricing of credit derivatives or derivatives on tax-exempt fixed income securities. In the case of credit derivatives, for instance, the pricing of options on defaultable bonds, for example, would ideally require the modelling of a two-factor risk-free rate process and a credit spread. Given the efficiency of the two-factor model presented here, it should be possible to approximate such a threefactor model, at least for a limited number of time periods. This is a subject for further research. 


\section{References}

1. Andersen, L. (2000), "A Simple Approach to the Pricing of Bermudan Swaptions," Journal of Computational Finance, 3, 5-32.

2. Andersen, L. and Andreasen (2001), "Factor Dependence of Bermudan Swaptions: Fact or Fiction?" Journal of Financial Economics, , 62, 3-38.

3. Balduzzi, P., S.R.Das, and S. Foresi (1998), "The Central Tendency: A Second Factor in Bond Yields," The Review of Economics and Statistics, 80, 62-72.

4. Black, F., E. Derman, and W. Toy (1990), "A One-Factor Model of Interest Rates and its Application to Treasury Bond Options," Financial Analysts' Journal, 46, 33-39.

5. Black, F. and P. Karasinski (1991), "Bond and Option Pricing when Short Rates are Lognormal," Financial Analysts Journal, 47, 52-59

6. Brace, A., D. Gatarek, and M. Musiela, (1997), "The Market Model of Interest Rate Dynamics," Mathematical Finance, 7, 127-155.

7. Brennan, M.J. and E.S. Schwartz (1979), "A Continuous-Time Approach to the Pricing of Bonds," Journal of Banking and Finance, 3, 135-155.

8. Brennan,M.J. and E.S. Schwartz (1982), "An Equilibrium Model of Bond Pricing and a Test of Market efficiency," Journal of Financial and Quantitative Analysis, 17, $301-329$.

9. Courtadon, G. (1982), "The Pricing of Options on Default-Free Bonds," Journal of Financial and Quantitative Analysis, 17, 75-100.

10. Cox, J.C., J.E. Ingersoll, and S.A. Ross (1981), "The Relationship between Forward Prices and Futures Prices," Journal of Financial Economics, 9, 1981, 321-46.

11. Dai, Q. and Singleton, K.J., (2000) "Specification Analysis of Affine Term Structure," Journal of Finance, 55, 1943-78.

12. Gong, F. and E.M. Remolona, (1997) "Two Factors along the Yield Curve," The Manchester School Supplement,65, 1-31.

13. Gupta, A. and M.G. Subrahmanyam (2000), "An Empirical Examination of the Convexity Bias in the Pricing of Interest Rate Swaps," Journal of Financial Economics, 55, 239-279. 
14. Heath, D. (1998), "Some New Term Structure Models," working paper, Carnegie Mellon University

15. Heath, D., R.A. Jarrow, and A. Morton (1992), "Bond Pricing and the Term Structure of Interest Rates: A New Methodology for Contingent Claims Valuation," Econometrica, 60, 1, January, 77-105.

16. Ho, T.S.Y. and S.B. Lee (1986), "Term Structure Movements and Pricing of Interest Rate Claims," Journal of Finance, 41, December, 1011-1029.

17. Ho. T.S., R.C. Stapleton, and M.G. Subrahmanyam (1995), "Multivariate Binomial Approximations for Asset Prices with Non- Stationary Variance and Covariance Characteristics," Review of Financial Studies, 8, 1125-1152.

18. Hull, J. and A. White (1994), "Numerical Procedures for Implementing Term Structure Models II: Two-Factor Models," Journal of Derivatives, 2, 37-48.

19. Hull, J. and A. White (2000), "Forward Rate Volatilities, Swap Rate Volatilities and the Implementation of the Libor Market Model," Working Paper, University of Toronto.

20. Jamshidian, F. (1989), "An Exact Bond Option Formula," Journal of Finance, 44, 205-209.

21. Li, A., P. Ritchken and L. Sankasubramanian (1995), "Lattice Models for Pricing American Interest Rate Claims," Journal of Finance, 50, 55-72

22. Longstaff, F., P. Santa-Clara, and E.S. Schwartz (2000), "The Relative Value of Caps and Swaptions: Theory and Empirical Evidence," UCLA Working paper.

23. Longstaff, F., P. Santa-Clara, and E.S. Schwartz (2001), "Throwing Away a Billion Dollars: The Cost of Suboptimal Exercise Strategies in the Swaptions Market," Journal of Financial Economics, , 62, 39-66.

24. Moraleda, J.M. and A. Pelsser (2000), "Forward versus Spot Interest Rate Models of the Term Structure: An Empirical Comparison," The Journal of Derivatives, Spring, $9-21$.

25. Miltersen, K.R., K. Sandmann and D. Sondermann (1997), "Closed Form Solutions for Term Structure Derivatives with Log-Normal Interest Rates," Journal of Finance, 1, 409-430.

26. Nelson, D.B. and K. Ramaswamy (1990), "Simple Binomial Processes as Diffusion Approximations in Financial Models," Review of Financial Studies, 3, 393-430. 
27. Peterson, S.J. (1999), "The Application of Binomial Trees to Calculate Complex Option Prices, Two-factor Stochastic Interest Rate Option Prices and Value-at-Risk," Ph.D Thesis, Lancaster University.

28. Peterson, S.J. and R.C. Stapleton (2000), "The Valuation of Options on Correlated Assets," Working paper, University of Strathclyde.

29. Pliska, S. Introduction to Mathematical Finance: Discrete Time Models, Oxford: Blackwell, 1997.

30. Rebonato, R. (1999), "On the Simultaneous Calibration of Multifactor Lognormal Interest Rate Models to Black Volatilities and to the Correlation Matrix" Journal of Computational Finance, 4, 5-27.

31. Rebonato, R. and I. Cooper (1995), "Limitations of simple two-factor interest rate models," Journal of Financial Engineering, 5, 1-16.

32. Ritchken, P. and L. Sankasubramanian (1995), "Volatility Structures of Forward Rates and the Dynamics ofthe Term Structure," Mathematical Finance, 5, 55-72.

33. Schmidt, J.W. (1968), "Asymptotische Einschliebung bei Konvergengenzbeschlenigenden Verfahren. II," Numerical Mathematics, 11, 53-56.

34. Sidenius, J. (2000), "LIBOR Market Models in Practice," Journal of Computational Finance, 3, 5-26.

35. Stapleton, R.C. and M.G. Subrahmanyam (2001) "The Term Structure of InterestRate Futures Prices," working paper, New York University.

36. Sundaresan, S. (1991), "Futures Prices on Yields, Forward Prices, and Implied Forward Prices from Term Structure," Journal of Financial and Quantitative Analysis, 26, 409-424.

37. Vasicek, O., (1977), "An Equilibrium Characterization of the Term Structure," Journal of Financial Economics, 5, 177-188. 
The Valuation of Caps, Floors and Swaptions

Table 1: Computing Time for Bond and Option Pricing (seconds)

\begin{tabular}{lccccc}
\hline Number of Periods & $\mathbf{8}$ & $\mathbf{1 2}$ & $\mathbf{2 4}$ & $\mathbf{4 8}$ & $\mathbf{7 2}$ \\
\hline & & & & & \\
Binomial Density 1 & 0.1 & 0.2 & 0.9 & 4.8 & 17.2 \\
Binomial Density 2 & 0.2 & 0.6 & 5.0 & 28.0 & 102.9 \\
Binomial Density 3 & 0.6 & 1.7 & 14.8 & 87.0 & - \\
& & & & & \\
\hline
\end{tabular}

The table shows the time taken to compute all the zero-bond prices, swaption prices, given the tree of rates for different levels of the binomial density, i.e., the number of time steps per period, for different numbers of periods. The computer speed is $550 \mathrm{MHZ}$, and the processor is Pentium III. 
The Valuation of Caps, Floors and Swaptions

Table 2: Caplet Volatility Matrix on July 18, 2000 (\%)

\begin{tabular}{|c|c|c|c|c|c|c|}
\hline \multirow[b]{2}{*}{ Maturity } & \multicolumn{3}{|c|}{ Strike Rate } & \multirow[b]{2}{*}{$6.5 \%$} & \multirow[b]{2}{*}{$7.0 \%$} & \multirow[b]{2}{*}{$8.0 \%$} \\
\hline & $5.0 \%$ & $5.5 \%$ & $6.0 \%$ & & & \\
\hline 3 & 15.86 & 14.86 & 13.86 & 11.33 & 8.80 & 9.73 \\
\hline 12 & 19.03 & 16.60 & 14.86 & 13.74 & 12.63 & 12.73 \\
\hline 18 & 19.60 & 17.15 & 15.67 & 15.15 & 14.63 & 14.39 \\
\hline 36 & 18.01 & 16.50 & 16.00 & 16.00 & 16.00 & 16.30 \\
\hline 60 & 16.80 & 15.80 & 14.80 & 14.80 & 14.80 & 15.00 \\
\hline 84 & 16.10 & 15.10 & 14.30 & 14.30 & 14.30 & 14.30 \\
\hline 120 & 15.80 & 14.90 & 13.90 & 13.85 & 13.80 & 13.80 \\
\hline
\end{tabular}

1. The Table shows the mid-market quotes (Black implied volatilities) for US\$ caplets, for different strike rates, quoted on July 18, 2000 in per cent.

2. Source: Sanwa International plc.

Table 3: Interpolated Futures Rates on July 18, 2000 (\%)

\begin{tabular}{ccccc}
\hline Maturity Year & Jan & April & July & Oct \\
\hline & & & & \\
2000 & & & & 7.02 \\
2001 & 6.98 & 7.11 & 7.14 & 7.18 \\
2002 & 7.16 & 7.15 & 7.16 & 7.19 \\
2003 & 7.18 & 7.18 & 7.20 & 7.25 \\
2004 & 7.25 & 7.26 & 7.29 & 7.36 \\
2005 & 7.36 & 7.37 & 7.41 & 7.47 \\
2006 & 7.48 & 7.49 & 7.52 & 7.59 \\
2007 & 7.60 & 7.61 & 7.64 & 7.70 \\
2008 & 7.71 & 7.72 & 7.76 & 7.82 \\
2009 & 7.82 & 7.83 & 7.87 & 7.93 \\
2010 & 7.94 & 7.95 & & \\
& & & & \\
\hline
\end{tabular}

1. The futures rates are derived by interpolation between adjacent contracts from Eurodollar futures prices. Futures rates are defined as 100-futures price in per cent.

2. Source: Bloomberg. 
The Valuation of Caps, Floors and Swaptions

Table 4 : At-the-money Caplet Black Volatilities on July 18, 2000 (\%)

\begin{tabular}{cccc}
\hline Maturity & Market Vols & Model 1 Vols & Model 2 Vols \\
\hline 3 & 8.80 & 9.3 & 9.8 \\
12 & 12.63 & 12.7 & 14.3 \\
18 & 14.63 & 14.6 & 15.4 \\
36 & 16.00 & 15.9 & 15.7 \\
60 & 14.80 & 15.1 & 15.0 \\
84 & 14.30 & 13.9 & 14.1 \\
RMSE(\%) & & 0.21 & 0.87 \\
& & & \\
\hline
\end{tabular}

1. Column 2 shows the prices of European-style caplets, quoted as Black implied volatilities.

2. Column 3 shows the corresponding prices produced by the two-factor model with parameter values $\sigma_{r}=0.099, b=1.7, \sigma_{\pi}=0.092$, and $c=0.13$. The model prices are estimated by Richardson extrapolation, using a binomial density of $n=3$ and $n=4$.

3. Column 4 shows the corresponding prices produced by the two-factor model with parameter values $\sigma_{r}=0.098, b=2.65, \sigma_{\pi}=0.1225$, and $c=0.087$. The model prices are computed using a binomial density of $n=2$.

Table 5: Market Swaption Black Volatility Quotes on July 18, 2000 (\%)

\begin{tabular}{cccccc}
\hline $\begin{array}{c}\text { Option } \\
\text { Maturity }\end{array}$ & $\begin{array}{c}\text { Swap } \\
12\end{array}$ & $\begin{array}{c}\text { Maturity } \\
24\end{array}$ & 36 & 48 & 60 \\
\hline & & & & & \\
12 & 13.29 & 13.80 & 13.70 & 13.60 & 13.50 \\
24 & 14.98 & 14.49 & 14.10 & 13.90 & 13.70 \\
36 & 15.00 & 14.50 & 14.10 & 13.82 & 13.70 \\
48 & 14.90 & 14.30 & 13.90 & 13.69 & 13.50 \\
60 & 14.70 & 14.20 & 13.90 & 13.60 & 13.30 \\
84 & 13.20 & 12.60 & 12.20 & 12.00 & 11.80 \\
& & & & & \\
\hline
\end{tabular}

1. Prices of European-style at-the-money US $\$$ swaptions quoted as Black implied volatilities on July 18, 2000 in per cent.

2. Source: Sanwa International plc. 
The Valuation of Caps, Floors and Swaptions

Table 6 : Two-Factor Model Swaption Volatilities for July 18, 2000 (\$)

\begin{tabular}{cccccc}
\hline Option & Swap & Maturity & & & \\
Maturity & 12 & 24 & 36 & 48 & 60 \\
\hline \multirow{2}{*}{12} & 15.42 & 16.60 & 16.61 & 16.11 & 15.48 \\
& $(2.13)$ & $(2.80)$ & $(2.91)$ & $(2.51)$ & $(1.98)$ \\
24 & 16.84 & 16.96 & 16.46 & 15.76 & 15.05 \\
& $(1.86)$ & $(2.47)$ & $(2.36)$ & $(1.86)$ & $(1.35)$ \\
36 & 16.69 & 16.44 & 15.82 & 15.07 & 14.33 \\
& $(1.69)$ & $(1.94)$ & $(1.72)$ & $(1.25)$ & $(0.63)$ \\
48 & 16.12 & 15.70 & 15.04 & 14.31 & 13.59 \\
& $(1.22)$ & $(1.40)$ & $(1.14)$ & $(0.62)$ & $(0.09)$ \\
60 & 15.40 & 14.96 & 14.30 & 13.57 & 12.86 \\
& $(0.70)$ & $(0.76)$ & $(0.40)$ & $(-0.03)$ & $(-0.44)$ \\
& & & & & \\
\hline
\end{tabular}

1. Prices of European-style swaptions quoted as Black implied volatilities in per cent. All swaption prices are computed using Richardson extrapolation, given model prices computed using the two-factor model with binomial densities $n=2$ and $n=3$.

2. The numbers in brackets are the differences between the model prices and the market quotes (i.e. model prices minus market quotes), where the market quotes are shown in Table 5.

3. The model optimal parameters are chosen to mimise the errors from fitting the model to cap volatility quotes. The parameter values are $\sigma_{r}=0.099, b=1.7, \sigma_{\pi}=0.092$, and $c=0.13$.

4. The RMSE for the swaption volatilities is $1.67 \%$. 
Table 7 : Three-Factor Model Swaption Volatilities for July 18, 2000 (\%)

\begin{tabular}{cccccc}
\hline $\begin{array}{c}\text { Option } \\
\text { Maturity }\end{array}$ & $\begin{array}{c}\text { Swap } \\
12\end{array}$ & $\begin{array}{c}\text { Maturity } \\
24\end{array}$ & 36 & 48 & 60 \\
\hline \multirow{2}{*}{12} & 14.68 & 15.85 & 15.84 & 15.44 & 14.90 \\
& $(1.39)$ & $(2.05)$ & $(2.14)$ & $(1.84)$ & $(1.40)$ \\
24 & 15.24 & 15.44 & 15.01 & 14.45 & 13.86 \\
& $(0.26)$ & $(0.95)$ & $(0.91)$ & $(0.55)$ & $(0.16)$ \\
36 & 14.90 & 14.80 & 14.29 & 13.70 & 13.12 \\
& $(-0.10)$ & $(0.30)$ & $(0.19)$ & $(-0.12)$ & $(-0.58)$ \\
48 & 14.36 & 14.15 & 13.63 & 13.06 & 12.49 \\
& $(-0.50)$ & $(-0.20)$ & $(-0.30)$ & $(-0.60)$ & $(-1.00)$ \\
60 & 13.83 & 13.54 & 13.04 & 12.48 & 11.95 \\
& $(-0.87)$ & $(-0.66)$ & $(-0.86)$ & $(-1.12)$ & $(-1.35)$ \\
& & & & & \\
\hline
\end{tabular}

1. Prices of European-style swaptions quoted as Black implied volatilities in per cent. All swaption prices are computed using Richardson extrapolation, given model prices computed using the three-factor model with binomial densities $n=2$ and $n=3$.

2. The numbers in brackets are the differences between the model prices and the market quotes (themselves shown in Table 5).

3. The model parameters are $\sigma_{r}=0.099, b=1.7, \sigma_{\pi}=0.092, c=0.13$, and $\sigma_{z}=0.07$.

4. The RMSE for the swaption volatilities is $0.98 \%$ 
Table 8: European and Bermudan Swaption Prices: Model Calibrated to Caplet Volatilities on July 18, 2000 (Basis Points)

\begin{tabular}{lccccccc}
\hline & & \multicolumn{5}{c}{ European } & \\
& Strike & One & year & option & on & (years) & Bermudan \\
\cline { 3 - 6 } & Rate & 1 & 2 & 3 & 4 & 5 & 6 \\
\hline \multirow{2}{*}{ Factor } & $6.5 \%$ & 80 & 158 & 231 & 300 & 365 & 414 \\
$n=2$ & $7.5 \%$ & 28 & 62 & 92 & 119 & 142 & 220 \\
& $8.5 \%$ & 6 & 17 & 26 & 34 & 39 & 115 \\
& & & & & & & \\
2 Factor & $6.5 \%$ & 82 & 161 & 235 & 304 & 371 & 421 \\
$n=3$ & $7.5 \%$ & 30 & 65 & 96 & 122 & 145 & 228 \\
& $8.5 \%$ & 8 & 19 & 30 & 36 & 41 & 122 \\
2 Factor & $6.5 \%$ & 84 & 164 & 239 & 308 & 377 & 428 \\
RE & $7.5 \%$ & 32 & 68 & 100 & 125 & 148 & 236 \\
& $8.5 \%$ & 10 & 21 & 34 & 38 & 43 & 129 \\
& & & & & & & \\
\hline \multirow{3}{3}{ Factor } & $6.5 \%$ & 77 & 151 & 221 & 289 & 356 & 389 \\
$n=2$ & $7.5 \%$ & 24 & 53 & 79 & 103 & 126 & 187 \\
& $8.5 \%$ & 4 & 11 & 17 & 22 & 26 & 84 \\
3 Factor & $6.5 \%$ & 78 & 154 & 223 & 287 & 349 & 387 \\
$n=3$ & $7.5 \%$ & 27 & 57 & 83 & 106 & 126 & 190 \\
& $8.5 \%$ & 5 & 14 & 21 & 26 & 30 & 90 \\
RE Factor & $6.5 \%$ & 79 & 157 & 225 & 285 & 342 & 385 \\
& $7.5 \%$ & 30 & 61 & 87 & 109 & 126 & 193 \\
& $8.5 \%$ & 6 & 17 & 25 & 30 & 34 & 96 \\
\hline
\end{tabular}

1. The above table shows the values of European and Bermurdan payer swaptions (in basis points) at differing depths in-the-money, when the two-factor model is calibrated to the caplet and futures data in Tables 2 and 3. The European swaptions are 1-year options on 1-year to 5 -year swaps. The Bermudan swaption is exercisable yearly for 5 years on a swap that terminates at the end of year 6 .

2. The model parameters are $\sigma_{r}=0.099, b=1.7, \sigma_{\pi}=0.092, c=0.13$ in the case of the two-factor model and $\sigma_{r}=0.099, b=1.7, \sigma_{\pi}=0.092, c=0.13$ and $\sigma_{z}=0.07$ in the case of 
the three-factor model.

3. RE refers to the estimate made by Richardson Extrapolation using the $n=2$ and $n=3$ estimates. 
Table 9 : Valuation of Bermudan and European Yield Spread Options: Model Calibrated to Caplet Volatilities on July 18, 2000 (Basis Points)

\begin{tabular}{ccccc}
\hline & European & \multicolumn{3}{c}{ Bermudan } \\
\hline 2-Factor Model & & & & \\
Strike (\%) & 0 & 0.50 & 0 & 0.50 \\
\hline$n=2$ & 20.0 & 3.2 & 32.3 & 6.1 \\
$n=3$ & 20.0 & 2.8 & 31.6 & 5.2 \\
$n=4$ & 20.0 & 2.5 & 31.2 & 4.7 \\
RE & 20.0 & 2.3 & 30.8 & 4.1 \\
\hline & & & & \\
3-Factor Model & & & & \\
Strike (\%) & 0 & 0.50 & 0 & 0.50 \\
\hline$n=2$ & 20.2 & 2.9 & 32.3 & 5.4 \\
$n=3$ & 18.9 & 2.7 & 31.0 & 5.1 \\
RE & 17.6 & 2.4 & 29.7 & 4.7 \\
\hline
\end{tabular}

1. The table shows the price (in basis points) of European-style and Bermudan-style call options on the spread between the four-year and the one-year zero-coupon bond yields.

2. Prices are estimated using binomial densities of of $n=2,3,4$. RE refers to the estimate made by Richardson Extrapolation using the $n=3$ and $n=4$ estimates in the case of the two-factor model, and using the $n=2$ and $n=3$ estimates in the case of the three-factor model.

3. The model parameters are $\sigma_{r}=0.099, b=1.7, \sigma_{\pi}=0.092, c=0.13$ in the case of the two-factor model and $\sigma_{r}=0.099, b=1.7, \sigma_{\pi}=0.092, c=0.13$ and $\sigma_{z}=0.07$ in the case of the three-factor model. 
Table 10 : Two-Factor Model Calibrated to Cap and Swaption Volatilities for July 18, 2000 (\%)

\begin{tabular}{|c|c|c|c|c|c|}
\hline Option & & Swap & Maturity & & \\
\hline Maturity & 12 & 24 & 36 & 48 & 60 \\
\hline 12 & $\begin{array}{l}13.84 \\
(0.55)\end{array}$ & $\begin{array}{l}14.96 \\
(1.16)\end{array}$ & $\begin{array}{l}15.11 \\
(1.41)\end{array}$ & $\begin{array}{c}14.88 \\
(1.28)\end{array}$ & $\begin{array}{l}14.49 \\
(0.99)\end{array}$ \\
\hline 24 & $\begin{array}{c}14.98 \\
(0.00)\end{array}$ & $\begin{array}{l}15.28 \\
(0.79)\end{array}$ & $\begin{array}{l}15.10 \\
(1.00)\end{array}$ & $\begin{array}{c}14.71 \\
(0.81)\end{array}$ & $\begin{array}{l}14.25 \\
(0.55)\end{array}$ \\
\hline 36 & $\begin{array}{c}15.02 \\
(0.02)\end{array}$ & $\begin{array}{c}15.04 \\
(0.54)\end{array}$ & $\begin{array}{l}14.73 \\
(0.63)\end{array}$ & $\begin{array}{l}14.28 \\
(0.46)\end{array}$ & $\begin{array}{c}13.79 \\
(0.09)\end{array}$ \\
\hline 48 & $\begin{array}{c}14.74 \\
(-0.16)\end{array}$ & $\begin{array}{l}14.63 \\
(0.33)\end{array}$ & $\begin{array}{l}14.26 \\
(0.36)\end{array}$ & $\begin{array}{c}13.79 \\
(0.10)\end{array}$ & $\begin{array}{c}13.30 \\
(-0.20)\end{array}$ \\
\hline 60 & $\begin{array}{c}14.34 \\
(-0.36)\end{array}$ & $\begin{array}{c}14.17 \\
(-0.03)\end{array}$ & $\begin{array}{c}13.77 \\
(-0.13)\end{array}$ & $\begin{array}{c}13.29 \\
(-0.31)\end{array}$ & $\begin{array}{c}12.80 \\
(-0.50)\end{array}$ \\
\hline
\end{tabular}

1. Prices of European-style swaptions quoted as Black implied volatilities in per cent. All swaption prices are computed using the two-factor model with a binomial density of $n=2$. The model parameters are chosen to minimise the difference between caplet and swaption volatilities, with a weight of 0.75 on caplets and 0.25 on swaptions. The best fit parameters are $\sigma_{r}=0.098, b=2.65, \sigma_{\pi}=0.1225$, and $c=0.087$.

2. In brackets are the differences between the model prices and the market quotes (as shown in Table 5) in per cent.

3. The RMSE of the swaption volatilities is $0.65 \%$. 
The Valuation of Caps, Floors and Swaptions

Table 11: Swaption Prices Calibrated to Market Volatilities on July 18, 2000 (Basis Points)

\begin{tabular}{cccccccc}
\hline & & \multicolumn{6}{c}{ European } \\
& Strike & One & year & option & on & (years) & Bermudan \\
\cline { 3 - 6 } & Rate & 1 & 2 & 3 & 4 & 5 & 6 \\
\hline \multirow{3}{*}{2 factor } & $6.5 \%$ & 79 & 156 & 228 & 296 & 363 & 407 \\
& $7.5 \%$ & 28 & 59 & 87 & 113 & 136 & 211 \\
& $8.5 \%$ & 6 & 15 & 23 & 30 & 36 & 107 \\
\hline
\end{tabular}

1. The above table shows the values (in basis points) of European-style and Bermurdan-style payer swaptions at differing depths in-the-money, when the two-factor model is calibrated to both the caplets and swaption data in Tables 2 and 5. The European swaptions are 1-year options on 1-year to 5-year swaps. The Bermudan swaption is exercisable yearly for 5 on a swap with final maturity at year 6 .

2. The 2 -factor model is the model where the current short rate is $7 \%$, the futures rate curve is described in Table 3, the conditional short-rate volatility of $9.8 \%$, with a coefficient of mean reversion of the short rate of $265 \%$, and volatility of the premium at $12.25 \%$ with $8.7 \%$ coefficient of mean reversion. The model uses a binomial density of $n=2$. 


\section{Appendices}

\section{A Proof of Lemma 1}

The price of the futures $L I B O R$ contract is by definition

$$
F_{t, T}=1-f_{t, T}
$$

and its price at maturity is

$$
F_{T, T}=1-f_{T, T}=1-r_{T} .
$$

From Cox, Ingersoll and Ross (1981), the futures price $F_{t, T}$ is the value, at time $t$, of an asset that pays

$$
V_{T}=\frac{1-r_{T}}{B_{t, t+1} B_{t+1, t+2} \ldots B_{T-1, T}}
$$

at time $T$, where the time period from $t$ to $t+1$ is one day. In a no-arbitrage economy, there exists a risk-neutral measure, under which the time- $t$ value of the payoff is

$$
F_{t, T}=E_{t}\left(V_{T} B_{t, t+1} B_{t+1, t+2} \ldots B_{T-1, T}\right) .
$$

Substituting (16) in (17), and simplifying then yields

$$
F_{t, T}=E_{t}\left(1-r_{T}\right)=1-E_{t}\left(r_{T}\right) .
$$

Combining (18) with (14) yields the first statement in the lemma. The second statement in the lemma follows from the assumption of the lognormal process for $r_{T}$ and the moment generating function of the normal distribution.

\section{B Proof of Lemma 2}

Taking the unconditional expectation of equation (1),

$$
\begin{gathered}
\mu_{r_{t}}-\mu_{r_{t-1}}=\theta_{r_{t}}-b \mu_{r_{t-1}}+\mu_{\pi_{t-1}}, \\
\mu_{\pi_{t}}-\mu_{\pi_{t-1}}=\theta_{\pi_{t}}-c \mu_{\pi_{t-1}}+\mu_{z_{t-1}} \\
\mu_{z_{t}}-\mu_{z_{t-1}}=\theta_{z_{t}}-d \mu_{z_{t-1}}
\end{gathered}
$$


Then, substituting for $\theta_{r_{t}}, \theta_{\pi_{t}}$ and $\theta_{z_{t}}$ in (1) yields

$$
\begin{gathered}
\ln \left(r_{t}\right)-\mu_{r_{t}}=\left[\ln \left(r_{t-1}\right)-\mu_{r_{t-1}}\right](1-b)+\ln \left(\pi_{t-1}\right)-\mu_{\pi_{t-1}}+\varepsilon_{t} \\
\ln \left(\pi_{t}\right)-\mu_{\pi_{t}}=\left[\ln \left(\pi_{t-1}\right)-\mu_{\pi_{t-1}}\right](1-c)+\ln \left(z_{t-1}\right)-\mu_{z_{t-1}}+\nu_{t} \\
\ln \left(z_{t}\right)-\mu_{z_{t}}=\left[\ln \left(z_{t-1}\right)-\mu_{z_{t-1}}\right](1-d)+\eta_{t}
\end{gathered}
$$

Since $r_{t}, \pi_{t}$ and $z_{t}$ are lognormally distributed, it follows from the moment generating function of the normal distribution that

$$
\begin{aligned}
& E_{0}\left(r_{t}\right)=\exp \left(\mu_{r_{t}}+\sigma_{r_{t}}^{2} / 2\right) \\
& E_{0}\left(\pi_{t}\right)=\exp \left(\mu_{\pi_{t}}+\sigma_{\pi_{t}}^{2} / 2\right) \\
& E_{0}\left(z_{t}\right)=\exp \left(\mu_{z_{t}}+\sigma_{z_{t}}^{2} / 2\right)
\end{aligned}
$$

Lemma 1 then implies:

$$
\ln \left[f_{0, t}\right]=\ln \left[E_{0}\left(r_{t}\right)\right]=\mu_{r_{t}}+\sigma_{r_{t}}^{2} / 2
$$

and using $E\left(\pi_{t}\right)=1, E\left(z_{t}\right)=1$, we have

$$
\begin{aligned}
& \ln \left[E_{0}\left(\pi_{t}\right)\right]=0=\mu_{\pi_{t}}+\sigma_{\pi_{t}}^{2} / 2, \\
& \ln \left[E_{0}\left(z_{t}\right)\right]=0=\mu_{z_{t}}+\sigma_{z_{t}}^{2} / 2 .
\end{aligned}
$$

Substitution for $\mu_{r_{t}}, \mu_{\pi_{t}}$, and $\mu_{z_{t}}$ and then yields the statement in the lemma.

\section{Proof of Lemma 3}

From lemma 1, the no-arbitrage condition implies

$$
f_{t, t+1}=E_{t}\left(r_{t+1}\right)
$$

in all states and for all $t$. From the lognormality of $r_{t+1}$,

$$
E_{t}\left(r_{t+1}\right)=\exp \left\{E_{t}\left[\ln \left(r_{t+1}\right)\right]+\frac{\sigma(t+1)^{2}}{2}\right\} .
$$

Hence, the no-arbitrage condition requires

$$
\ln \left(f_{t, t+1}\right)=E_{t}\left[\ln \left(r_{t+1}\right)\right]+\frac{\sigma(t+1)^{2}}{2} .
$$


But, taking the expectation of equation (3), for $r_{t+1}$ yields:

$$
E_{t}\left[\ln \left(r_{t+1}\right)\right]=\ln \left(f_{0, t+1}\right)+\alpha_{r_{t+1}}+(1-b)\left[\ln \left(r_{t}\right)-\ln \left(f_{0, t}\right)\right]+\ln \pi_{t} .
$$

Hence, substituting (20) into (19) yields:

$$
\ln \left(f_{t, t+1}\right)=\ln \left(f_{0, t+1}\right)+\alpha_{r_{t+1}}+(1-b)\left[\ln \left(r_{t}\right)-\ln \left(f_{0, t}\right)\right]+\ln \pi_{t}+\frac{\sigma(t+1)^{2}}{2} .
$$

The lemma follows with

$$
\alpha_{f_{t+1}}=\alpha_{r_{t+1}}+\frac{\sigma(t+1)^{2}}{2} .
$$

This establishes the first part of the lemma. A similar argument can be used to prove the second part of the lemma.

\section{Proof of Proposition 2}

First, we derive the following covariances from equation (3)

$$
\begin{aligned}
\sigma_{r_{t+1}, r_{t}} & =(1-b) \sigma_{r_{t}}^{2}+\sigma_{r_{t}, \pi_{t}}, \\
\sigma_{r_{t}, \pi_{t}} & =(1-c) \sigma_{\pi_{t-1}}^{2}+(1-b)(1-c) \sigma_{r_{t-1}, \pi_{t-1}}+\operatorname{cov}\left(\varepsilon_{t}, \nu_{t}\right), \\
\sigma_{\pi_{t+1}, \pi_{t}} & =(1-c) \sigma_{\pi_{t}}^{2}, \\
\sigma_{r_{t}, \pi_{t-1}} & =(1-b) \sigma_{r_{t-1}, \pi_{t-1}}+\sigma_{\pi_{t-1}}^{2}, \\
\sigma_{r_{t-1}, \pi_{t}} & =(1-c) \sigma_{r_{t-1}, \pi_{t-1}}
\end{aligned}
$$

Now, from the multiple regression

$$
\ln \left[\frac{r_{t}}{f_{0, t}}\right]=\alpha_{r_{t}}+\beta_{r_{t}} \ln \left[\frac{r_{t-1}}{f_{0, t-1}}\right]+\gamma_{r_{t}} \ln \left(\pi_{t-1}\right)+\varepsilon_{t}
$$

the regression coefficients are

$$
\beta_{r_{t}}=\frac{\sigma_{r_{t}, r_{t-1}} \sigma_{\pi_{t-1}}^{2}-\sigma_{r_{t}, \pi_{t-1}} \sigma_{r_{t-1}, \pi_{t-1}}}{\sigma_{r_{t-1}}^{2} \sigma_{\pi_{t-1}}^{2}-\left(\sigma_{r_{t-1}, \pi_{t-1}}\right)^{2}}
$$




$$
\gamma_{r_{t}}=\frac{\sigma_{r_{t}, r_{t-1}} \sigma_{r_{t-1}}^{2}-\sigma_{r_{t}, r_{t-1}} \sigma_{r_{t-1}, \pi_{t-1}}}{\sigma_{r_{t-1}}^{2} \sigma_{\pi_{t-1}}^{2}-\left(\sigma_{r_{t-1}, \pi_{t-1}}\right)^{2}}
$$

Substituting the covariances and simplifying yields

$$
\beta_{r_{t}}=(1-b)
$$

and

$$
\gamma_{r_{t}}=1 .
$$

From the lognormality of $r_{t}$ and $\pi_{t}$ we can write equation (3) as

$$
\begin{aligned}
\ln \left(r_{t}\right)-\ln \left[f_{0, t}\right]+\sigma_{r_{t}}^{2} / 2 & =\left\{\ln \left(r_{t-1}\right)-\ln \left[f_{0, t-1}\right]+\sigma_{r_{t-1}}^{2} / 2\right\}(1-c) \\
& +\ln \left(\pi_{t-1}\right)-\left\{\sigma_{\pi_{t-1}}^{2} / 2\right\}+\varepsilon_{t} .
\end{aligned}
$$

Re-arranging terms yields

$$
\begin{aligned}
\ln \left[\frac{r_{t}}{f_{0, t}}\right] & =\left[-\sigma_{r_{t}}^{2}+\sigma_{r_{t}}^{2}(1-c)+\sigma_{\pi_{t}}^{2}\right] / 2 \\
& +\ln \left[\frac{r_{t-1}}{f_{0, t-1}}\right](1-c)+\ln \left(\pi_{t-1}\right)+\varepsilon_{t} .
\end{aligned}
$$

Given (21), (22), and (23), we have $\alpha_{r_{t}}$ as stated in the Proposition.

Similarly, with $E_{0}\left(\pi_{t}\right)=1$, we have

$$
\ln \left(\pi_{t}\right)=\alpha_{\pi_{t}}+\ln \left(\pi_{t-1}\right)(1-c)+\ln \left(z_{t-1}\right)+\nu_{t},
$$

and

$$
\begin{gathered}
\alpha_{\pi_{t}}=E_{0}\left[\ln \left(\pi_{t}\right)\right]-(1-c)\left[E_{0}\left[\ln \left(\pi_{t-1}\right)\right]+\left[E_{0}\left[\ln \left(z_{t-1}\right)\right]\right.\right. \\
\alpha_{\pi_{t}}=\left[-\sigma_{\pi_{t}}^{2}+(1-c) \sigma_{\pi_{t-1}}^{2}+\sigma_{z_{t-1}}^{2}\right] / 2
\end{gathered}
$$


For $z_{t}$ we have:

$$
\ln \left(z_{t}\right)=\alpha_{z_{t}}+\ln \left(z_{t-1}\right)(1-d)+\eta_{t}
$$

and

$$
\begin{gathered}
\alpha_{z_{t}}=E_{0}\left[\ln \left(z_{t}\right)\right]-(1-d)\left[E_{0}\left[\ln \left(z_{t-1}\right)\right]\right. \\
\alpha_{z_{t}}=\left[-\sigma_{z_{t}}^{2}+(1-d) \sigma_{z_{t-1}}^{2}\right] / 2
\end{gathered}
$$

Finally, the variance of $\varepsilon_{t}$, given $t$, is

$$
\begin{aligned}
\operatorname{var}_{t-1}\left(\varepsilon_{t}\right) & =\operatorname{var}_{0}\left\{\ln \left[\frac{r_{t}}{f_{0, t}}\right]\right\}-\beta_{r, t}^{2} \operatorname{var}_{0}\left\{\ln \left[\frac{r_{t-1}}{f_{0, t-1}}\right]\right\} \\
& -\gamma_{r_{t}}^{2} \operatorname{var}_{0}\left[\ln \left(\pi_{t-1}\right)\right]-\beta_{r_{t}} \gamma_{r_{t}} \operatorname{cov}\left[\ln \left(r_{t-1}\right), \ln \left(\pi_{t-1}\right)\right]
\end{aligned}
$$

or,

$$
\operatorname{var}_{t-1}\left(\varepsilon_{t}\right)=\sigma_{r_{t}}^{2}-(1-b)^{2} \sigma_{r_{t-1}}^{2}-\sigma_{\pi_{t-1}}^{2}-2(1-b) \sigma_{r_{t-1}, \pi_{t-1}}
$$

\section{E Proof of Lemma 4}

Taking the conditional expectation of equation (3) at $t$

$$
E_{t}\left[\ln \left(r_{t+1}\right)\right]-\ln \left(f_{0, t+1}=\alpha_{r_{t}}+\left[\ln \left(r_{t}\right)-\ln \left(f_{0, t}\right](1-b)+\ln \left(\pi_{t}\right) .\right.\right.
$$

Given $x_{t}=E_{t}\left(r_{t+1}\right)$ and using the lognormal property of $r_{t+1}$,

$$
\begin{aligned}
\ln \left(x_{t}\right) & =E_{t}\left[\ln \left(r_{t+1}\right)\right]+\sigma_{r}^{2}(t+1) / 2 \\
& =\sigma_{r}^{2}(t+1) / 2+\ln \left(f_{0, t+1}+\left[\ln \left(r_{t}\right)-\ln \left(f_{0, t}\right](1-b)+\left[\ln \left(\pi_{t-1}\right)\right](1-c)+\nu_{t}\right.\right.
\end{aligned}
$$

Hence

$$
\sigma_{x}^{2}(t)=\operatorname{var}_{t-1}\left[\ln \left(x_{t}\right)\right]=(1-b)^{2} \operatorname{var}_{t-1}\left[\ln \left(r_{t}\right)\right]+\operatorname{var}_{t-1}\left[\nu_{t}\right]
$$

or

$$
\sigma_{x}^{2}(t)=\sigma_{\pi}^{2}(t)+(1-b)^{2} \sigma_{r}^{2}(t)
$$

and the statement in the lemma follows. 
Figure 1: A Recombining Two-factor Process for the Short-term Interest Rate (Two-period case)

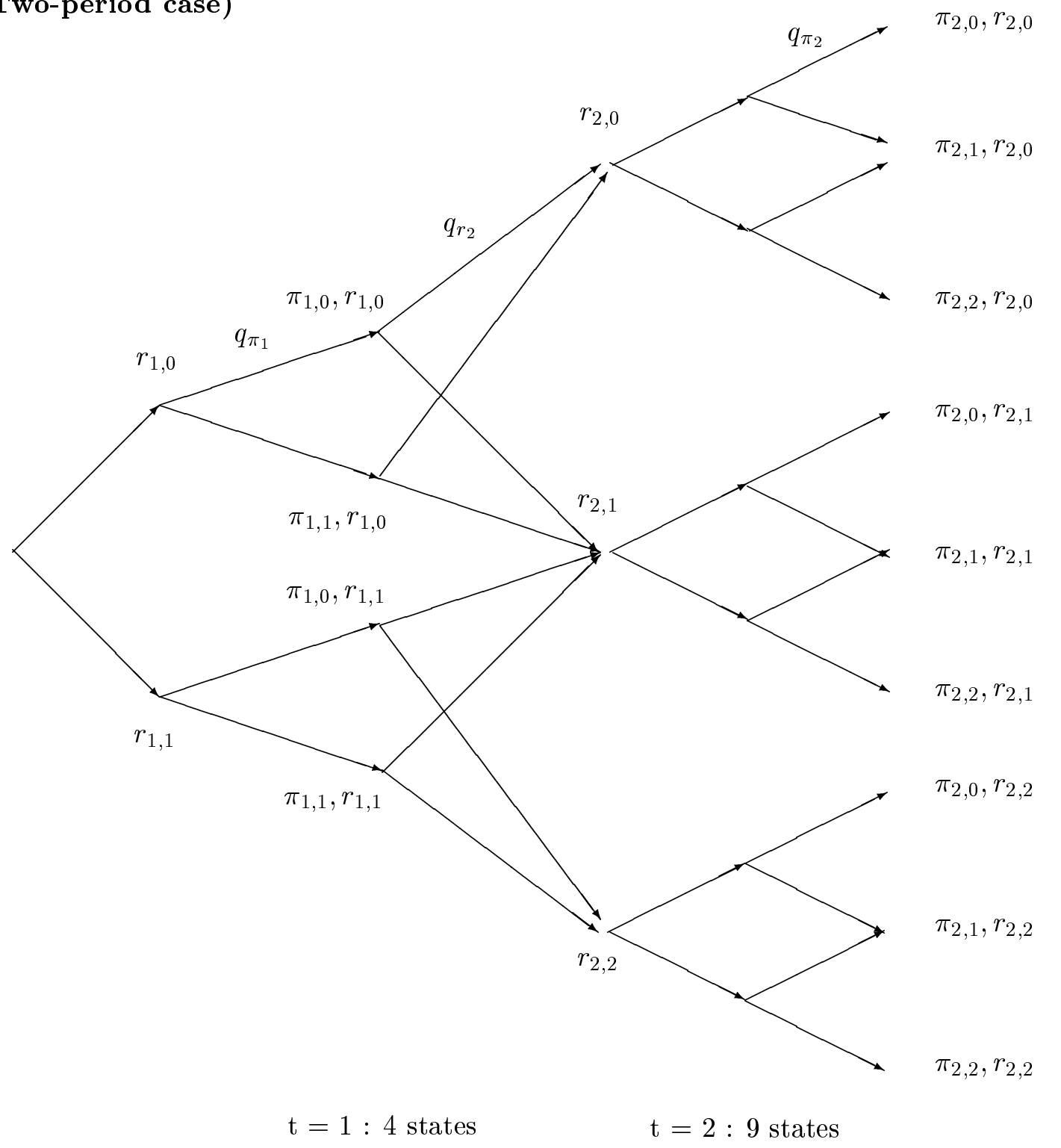

[1] The probability of moving, for example, to $r_{2,0}$ given $\left(\pi_{1,0}, r_{1,0}\right)$ is $q_{r_{2}}$, defined in Equation (11). [2] The probability of moving, for example, to $\left(\pi_{1,0}, r_{1,0}\right)$ given $r_{1,0}$ and $\pi_{1,0}$ is $q_{\pi_{1}}$, defined in Equation (10). 
Figure 2: A Recombining Two-factor Process for the Short-term Interest Rate (Three-period case)

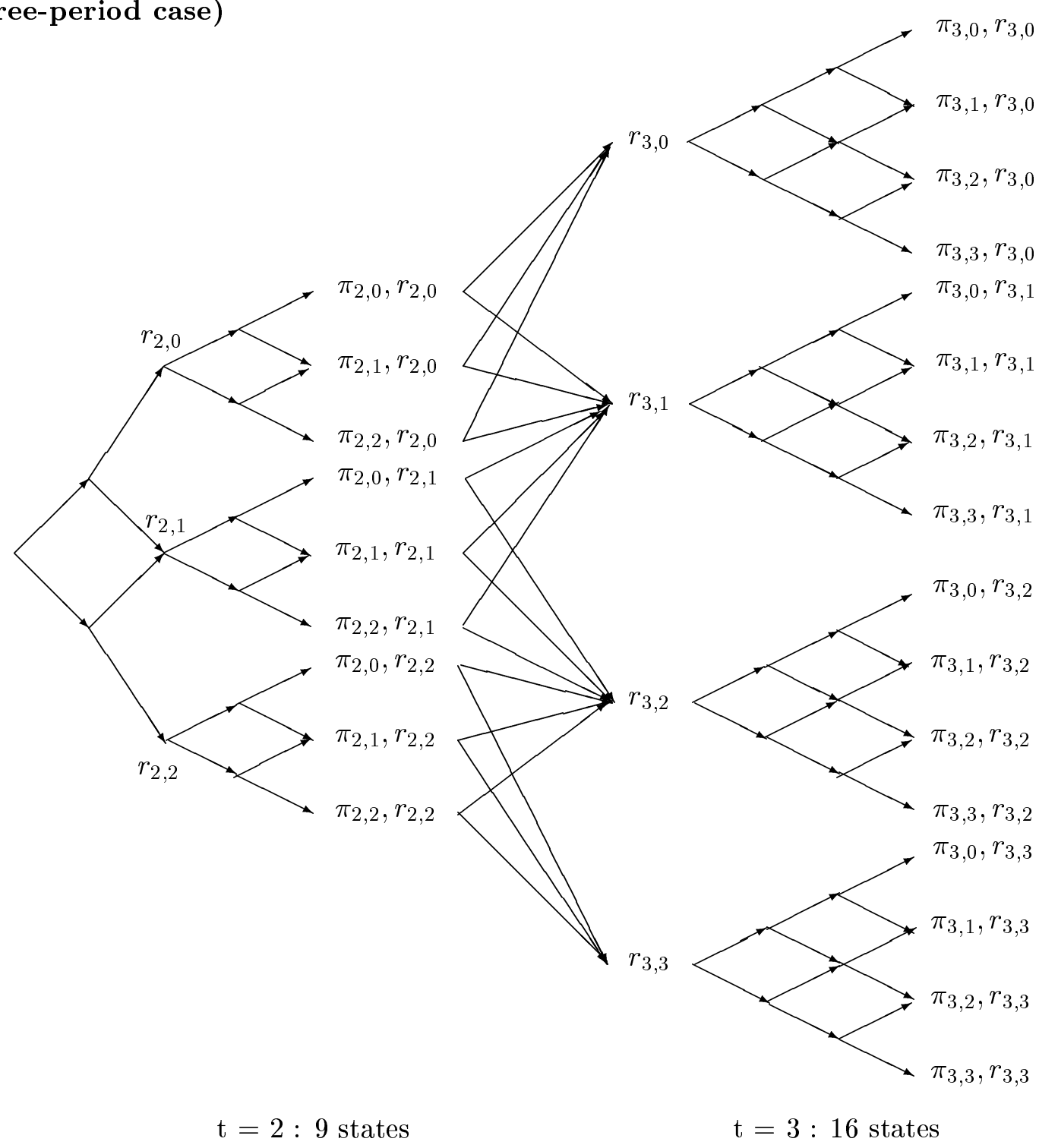

[1] The probability of moving, for example, to $r_{3,0}$ given $\left(\pi_{2,0}, r_{2,0}\right)$ is $q_{r_{2}}$, defined in Equation (11). [2] The probability of moving, for example, to $\left(\pi_{3,0}, r_{3,1}\right)$ given $r_{3,1}$ and $\pi_{2,0}$ is $q_{\pi_{3}}$, defined in Equation (10). 